The Astrophysical Journal, 267:571-595, 1983 April 15

(1) 1983. The American Astronomical Society. All rights reserved. Printed in U.S.A.

\title{
STELLAR ORBITS IN A TRIAXIAL GALAXY. I. ORBITS IN THE PLANE OF ROTATION
}

\author{
TIM DE ZEEUW \\ Sterrewacht, Huygens Laboratorium, Leiden \\ AND \\ DAVID MERRITT \\ Princeton University Observatory \\ Received 1982 February 26; accepted 1982 October 7
}

\begin{abstract}
An approximation technique for solving the equations of motion in nonintegrable potentials is applied to the case of stellar orbits in the plane of rotation of a nonaxisymmetric galaxy. The technique consists of $(a)$ expanding the potential about the origin up to quartic terms and $(b)$ replacing the corresponding Hamiltonian by a simpler averaged Hamiltonian, for which the equations of motion admit exact solutions. An explicit expression is obtained for the nonclassical second integral, and the major families of closed orbits are delineated.

The results of the analytical technique are compared with orbits calculated numerically in the plane of rotation of Schwarzschild's triaxial galaxy model. A resonance zone extends from a few core radii to infinity (or to roughly the Lindblad resonance if the galaxy is rotating); the structure of phase space in this zone is dominated by a set of 1:1 closed orbit families which do not exist in the harmonic (i.e., core) potential. It is suggested that most triaxial potentials are probably describable as 1:1:1 resonances, a result which has important implications for the existence of equilibrium triaxial galaxies.
\end{abstract}

Subject headings: galaxies: internal motions - galaxies: structure - stars: stellar dynamics

\section{INTRODUCTION}

It is by now generally agreed that the nonspherical shape of elliptical galaxies is not usually a result of flattening due to rotation about an axis of symmetry but must be caused by some other mechanism (Binney $1978 b$; Illingworth 1981). At least three lines of argument support this conclusion:

1. Measurements of rotation velocities (Illingworth 1977; Peterson 1978) and velocity dispersions (Schechter and Gunn 1979; Davies 1981) of the stars in elliptical galaxies show that the observed rotation is generally too small, by a large factor, to support the observed flattening. (The discrepancy tends to decrease with decreasing luminosity, however; see Davies et al. 1982.)

2. The apparent twisting on the sky of the isophotal brightness contours of many elliptical galaxies (King 1978; Williams and Schwarzschild 1979a, $b$; Leach 1981) is consistent with, and perhaps most easily explained by, these systems having triaxial density distributions.

3. A number of numerical investigations (Aarseth and Binney 1978; Miller 1978; Schwarzschild 1979, 1982) suggest that equilibrium triaxial systems are easy to construct, even in the absence of rotation, and that such systems can retain their shapes over many dynamical times (Wilkinson and James 1982; Smith and Miller 1982).

Simple numerical integration of the equations of motion in axisymmetric and triaxial potentials shows that the stellar orbits are often characterized by one or two isolating integrals in addition to the classical integrals, and this fact has been used in constructing self-consistent numerical models (Schwarzschild 1979, 1982; Richstone 1980; de Zeeuw et al. 1983). Indeed, it appears likely that these so-called nonclassical integrals are all that is needed in the general case to maintain elliptical galaxies in non-spherically-symmetric shapes. This point was first emphasized by Binney (1978a), who noted that a velocity distribution function that depends on more integrals than just the energy implies anisotropic velocity dispersions, which are required if an elliptical galaxy is to be supported without rotation. A similar situation has long been known to exist in our own galaxy, where the observed shape of the velocity ellipsoid near the Sun can best be explained by postulating a third isolating integral in addition to the energy and angular momentum (Jeans 1916; Contopoulos 1960; Ollongren 1962; Martinet and Mayer 1975). Although a few simple models for triaxial galaxies exist in which the stellar orbits are characterized by only the classical 
integrals (e.g., Vandervoort 1980), it seems unavoidable that nonclassical integrals play a crucial role in maintaining the shapes of most elliptical galaxies, especially the slowly rotating ones. This point is discussed further in $\S \mathrm{V}$.

Bulges of spiral galaxies are similar to elliptical galaxies in many respects. Although some of them may be triaxial (Stark 1977), it appears likely that most bulges are supported by rotation and not by anisotropic dispersions (Kormendy and Illingworth 1982).

The purpose of the present work is to investigate by analytical means some of the properties of stellar orbits in a nonaxisymmetric elliptical galaxy. By comparing the results so obtained to "actual" stellar orbits in a realistic model potential-that of Schwarzschild (1979) - we are able to shed some light on the way in which the nonclassical integrals affect the distribution of orbit types, and therefore the density distribution, in such models. It will turn out that the analytical technique, although formally valid only in the galaxy core, is very successful at predicting the nature of stellar orbits at larger energies. We will argue that this result is probably a general one for galactic potentials and not specific to Schwarzschild's model.

Although our method is capable of treating arbitrary orbits in a general three-dimensional potential, the present work is limited to a discussion of orbits in the plane of rotation. As pointed out by Vandervoort (1979), such orbits are particularly useful in understanding the more general case, which will be considered in subsequent papers. A few specific properties of threedimensional orbits in triaxial potentials have been described by Schwarzschild and collaborators (Heiligman and Schwarzschild 1979; Goodman and Schwarzschild 1981; Heisler, Merritt and Schwarzschild 1982), as well as by Magnenat (1982).

A number of studies have been made of planar orbits in nearly axisymmetric potentials, to simulate the bars of spiral galaxies (cf. Contopoulos 1975, 1978a; Contopoulos and Mertzanides 1977; Contopoulos and Papayannopoulos 1980). In contrast, our method is designed to treat systems which are inherently nonaxisymmetric (or "very strong bars" in the terminology of Contopoulos and Papayannopoulos) and which are not dominated by the circular or near-circular orbits characteristic of spiral galaxies. For this reason our method is well suited to studying systems with relatively little ordered motion, such as elliptical galaxies. Nevertheless there are numerous points of connection between our results and those of earlier workers, and these will be emphasized below.

In $\S$ II we give the relevant results of the analytical technique, the details of which are described elsewhere (de Zeeuw 1983). In $\S$ III we compare the analytical predictions for the integrals of motion and shapes of the periodic orbits to the orbits calculated numerically in
Schwarzschild's potential. In $\S$ IV we discuss the generality of the results obtained, and in $\S \mathrm{V}$ we note the implications for the existence of equilibrium triaxial galaxies.

\section{THEORY}

\section{a) Basic Equations}

Consider an idealized elliptical galaxy that rotates uniformly around a fixed axis with angular velocity $\Omega \geqslant 0$. Choose, in a corotating reference frame, Cartesian coordinates $(x, y, z)$ so that the $z$-axis coincides with the axis of rotation. The sign of $\Omega$ is such that the model rotates clockwise as seen from the positive $z$-axis. In what follows we will restrict ourselves to a study of the motion in the equatorial (i.e., $x-y$ ) plane.

Let $V^{*}(x, y)$ be the gravitational potential in the corotating frame. The Hamiltonian $H$ which determines the motion of a single star in this potential is

$$
H=\frac{1}{2} \dot{x}^{2}+\frac{1}{2} \dot{y}^{2}+V(x, y),
$$

where

$$
V(x, y) \equiv V^{*}(x, y)-\frac{1}{2} \Omega^{2}\left(x^{2}+y^{2}\right)
$$

The equations of motion are

$$
\begin{aligned}
& \ddot{x}=-2 \Omega \dot{y}-\partial V / \partial x, \\
& \ddot{y}=+2 \Omega \dot{x}-\partial V / \partial y .
\end{aligned}
$$

Since $V$ is assumed to be independent of time, the Hamiltonian is conserved. In other words, the total energy of a star moving in the gravitational potential, as measured in the corotating frame, is an integral of the motion. It is usually called "Jacobi's integral" (Jacobi 1836). In general, it is the only exact isolating integral of the motion known in rotating systems (Lynden-Bell 1962).

For potentials corresponding to elliptical galaxies, the equations of motion cannot be solved analytically, and one must resort to numerical methods in computing the stellar orbits. Perturbation techniques, although valid only in a restricted region of space and in most cases in a finite time interval, can nevertheless yield considerable insight into the properties of the orbits and of the integrals of motion which characterize them. A number of perturbation techniques are available (see, e.g., Hagihara 1974). For our purposes, the most useful is probably the method of asymptotic expansions based on obtaining Birkhoff normal forms by averaging procedures (Bogoliubov and Mitropolsky 1961; Van der Burgh 1974, 1976; Sanders and Verhulst 1981). In the present investigation we adopt this method, as described by Van der Burgh (1974) but with a slight modification, to study the behavior of orbits near the center where the poten- 
tial has a minimum and motion can be considered perturbed harmonic motion. A more detailed comparison between the present method and that of previous authors will be made in $\S \mathrm{V}$.

In this study we restrict ourselves to models that are symmetric with respect to the three principal planes, i.e., $V=V\left(x^{2}, y^{2}\right)$. In view of the regular shapes of elliptical galaxies, this should be a reasonable approximation. Effects of asymmetries are discussed briefly in $\S \mathrm{IV} c$.

\section{b) Linear Analysis}

Expanding the gravitational potential $V$ in a Taylor series about the origin gives

$$
\begin{aligned}
V(x, y)= & \frac{1}{2} \kappa_{1}{ }^{2} x^{2}+\frac{1}{2} \kappa_{2}{ }^{2} y^{2}+\frac{1}{4} a_{5} x^{4} \\
& +\frac{1}{2} a_{7} x^{2} y^{2}+\frac{1}{4} a_{9} y^{4}+\ldots,
\end{aligned}
$$

where it is understood that $0<\kappa_{1} \leqslant \kappa_{2}$. Retaining only the quadratic terms in the potential we find for the equations of motion of an individual star

$$
\begin{aligned}
& \ddot{x}=-2 \Omega \dot{y}-\kappa_{1}{ }^{2} x, \\
& \ddot{y}=+2 \Omega \dot{x}-\kappa_{2}{ }^{2} y .
\end{aligned}
$$

Equations (5) describe a two-dimensional uniformly rotating harmonic oscillator. The solutions of these equations have been discussed by Freeman (1966a; see also Hunter 1974), who investigated orbits in a uniform-density elliptical disk. We briefly summarize Freeman's main results and emphasize those aspects that are relevant to our investigation of the nonlinear equations.

The general solution of equations (5) is a linear combination of two uncoupled harmonic oscillations:

$$
\begin{aligned}
& x=A_{1} \cos \left(\omega_{1} t+\phi_{1}\right)+\alpha A_{2} \sin \left(\omega_{2} t+\phi_{2}\right), \\
& y=\beta A_{1} \sin \left(\omega_{1} t+\phi_{1}\right)+A_{2} \cos \left(\omega_{2} t+\phi_{2}\right),
\end{aligned}
$$

where $A_{1}, A_{2}, \phi_{1}$, and $\phi_{2}$ are constants determined by the initial conditions. The frequencies $\omega_{1}$ and $\omega_{2}$ are given by

$$
\begin{aligned}
\omega_{1,2}{ }^{2}= & \frac{1}{2}\left(\kappa_{1}{ }^{2}+\kappa_{2}{ }^{2}\right)+2 \Omega^{2} \\
& \mp \frac{1}{2}\left[\left(\kappa_{1}{ }^{2}+\kappa_{2}{ }^{2}+4 \Omega^{2}\right)^{2}-4 \kappa_{1}{ }^{2} \kappa_{2}{ }^{2}\right]^{1 / 2},
\end{aligned}
$$

and the constants $\alpha$ and $\beta$ are defined as

$$
\begin{aligned}
& \alpha \equiv 2 \Omega \omega_{2} /\left(\omega_{2}^{2}-\kappa_{1}^{2}\right)=\left(\omega_{2}^{2}-\kappa_{2}^{2}\right) / 2 \Omega \omega_{2}, \\
& \beta \equiv\left(\kappa_{1}{ }^{2}-\omega_{1}^{2}\right) / 2 \Omega \omega_{1}=2 \Omega \omega_{1} /\left(\kappa_{2}^{2}-\omega_{1}^{2}\right) .
\end{aligned}
$$

We note that $\Omega \neq 0$ implies $\omega_{1}<\omega_{2}$ even for $\kappa_{1}=\kappa_{2}$. Also,

$$
|\alpha| \leqslant 1, \quad|\beta| \leqslant 1 .
$$

The fundamental oscillations (normal modes) are given by $A_{1}=0$ and $A_{2}=0$, respectively. For $A_{2}=0$ the orbit is an ellipse with axial ratio $1:|\beta|$, with the major axis along the $x$-direction. The motion is direct (i.e., in the same direction as the rotation). For $A_{1}=0$ the orbit is an ellipse with axial ratio $|\alpha|: 1$, and with the major axis along the $y$-direction. The motion is retrograde. For $\Omega=0$ these closed orbits degenerate to straight lines along the $x$ - and $y$-axes, respectively. Closed orbits occur in the nonrotating case only when $\omega_{1} / \omega_{2}$ (i.e., $\kappa_{1} / \kappa_{2}$ ) is a rational number.

In the general case $\left(A_{1} \neq 0, A_{2} \neq 0\right)$, equations (6) show that the motion can be regarded as an elliptic epicycle, for which one of the normal modes provides the guiding center motion and the other the gyration around it.

The subsequent analysis of the nonlinear equations of motion is most easily carried out in terms of the actionangle variables $(\boldsymbol{I}, \boldsymbol{\theta})$ (Arnold 1978). In order to obtain these we first define normal variables $(\boldsymbol{Q}, \boldsymbol{P})$ by

$$
\begin{aligned}
Q_{1} & =(\sigma \beta)^{1 / 2}\left[\dot{y}+\left(\omega_{2} / \alpha\right) x\right] \\
& =(\beta / \sigma)^{1 / 2} A_{1} \cos \left(\omega_{1} t+\phi_{1}\right), \\
P_{1} & =(\sigma / \beta)^{1 / 2}\left[\dot{x}-\omega_{2} \alpha y\right] \\
& =-(\beta / \sigma)^{1 / 2} A_{1} \sin \left(\omega_{1} t+\phi_{1}\right), \\
Q_{2} & =(\sigma \alpha)^{1 / 2}\left[\dot{x}+\left(\omega_{1} / \beta\right) y\right] \\
& =(\alpha / \sigma)^{1 / 2} A_{2} \cos \left(\omega_{2} t+\phi_{2}\right), \\
P_{2} & =(\sigma / \alpha)^{1 / 2}\left[\dot{y}-\omega_{1} \beta x\right] \\
& =-(\alpha / \sigma)^{1 / 2} A_{2} \sin \left(\omega_{2} t+\phi_{2}\right),
\end{aligned}
$$

where the quantity $\sigma$ is defined as

$$
\sigma \equiv \frac{2 \Omega}{\omega_{2}^{2}-\omega_{1}^{2}}
$$

so that

$$
\sigma^{-1}=\frac{\omega_{2}}{\alpha}+\omega_{1} \beta=\omega_{2} \alpha+\frac{\omega_{1}}{\beta} .
$$

The second equality in each equation (10) is valid only in a harmonic potential. In normal variables the Hamiltonian $H^{(0)}$ corresponding to the linearized equations of 
motion becomes

$$
H^{(0)}=\frac{1}{2} \omega_{1}\left(P_{1}^{2}+Q_{1}^{2}\right)+\frac{1}{2} \omega_{2}\left(P_{2}^{2}+Q_{2}^{2}\right) .
$$

Action-angle variables $(I, \theta)$ are now obtained from the normal variables $(\boldsymbol{Q}, \boldsymbol{P})$ by the canonical transformation

$$
\begin{aligned}
Q_{i} & =\left(2 I_{i}\right)^{1 / 2} \cos \theta_{i}, \\
P_{i} & =-\left(2 I_{i}\right)^{1 / 2} \sin \theta_{i}, \quad i=1,2 .
\end{aligned}
$$

The Hamiltonian $H^{(0)}$ becomes, in action-angle variables,

$$
H^{(0)}=\omega_{1} I_{1}+\omega_{2} I_{2},
$$

so that

$$
\begin{aligned}
I_{i} & =E_{i} \propto A_{i}^{2}, \\
\theta_{i} & =\omega_{i} t+\phi_{i} .
\end{aligned}
$$

Here $E_{1}$ and $E_{2}$ are constants, and $\omega_{1} E_{1}$ and $\omega_{2} E_{2}$ are the energies, as measured in the rotating frame, associated with the guiding center drift and the superposed gyration. The normal modes are given simply by $I_{i}=0$. Evidently, for this case there are two independent isolating integrals of motion, $E_{1}$ and $E_{2}$. The two-dimensional rotating harmonic oscillator is therefore a simple example of a system which is characterized by one isolating integral in addition to the total energy.

\section{c) Nonlinear Analysis}

Rescale the coordinates such that $x=\varepsilon x^{*}, y=\varepsilon y^{*}$, where $\varepsilon$ is a small positive parameter. Omitting the asterisks after the transformation, and dividing $V$ by $\varepsilon^{2}$, we obtain from equation (4)

$$
\begin{aligned}
V= & \frac{1}{2} \kappa_{1}{ }^{2} x^{2}+\frac{1}{2} \kappa_{2}{ }^{2} y^{2} \\
& +\varepsilon^{2}\left(\frac{1}{4} a_{5} x^{4}+\frac{1}{2} a_{7} x^{2} y^{2}+\frac{1}{4} a_{9} y^{4}\right)+O\left(\varepsilon^{4}\right) .
\end{aligned}
$$

Expressing the corresponding Hamiltonian $H$ in terms of action-angle variables, we find

$$
H(\boldsymbol{I}, \boldsymbol{\theta})=H^{(0)}(\boldsymbol{I})+\varepsilon^{2} H_{4}(\boldsymbol{I}, \boldsymbol{\theta})+O\left(\varepsilon^{4}\right),
$$

where $H^{(0)}$ is given by equation (15) and

$$
\begin{aligned}
H_{4}= & \sum_{i, j, k, l} I_{1}^{|i| / 2} I_{2}^{|j| / 2}\left[\lambda_{i j k l} \cos \left(k \theta_{1}-l \theta_{2}\right)\right. \\
& \left.+\mu_{i j k l} \sin \left(k \theta_{1}-l \theta_{2}\right)\right] .
\end{aligned}
$$

Here $i$ and $j$ take all integer values satisfying

$$
|i|+|j|=4,
$$

and

$$
\begin{aligned}
k & =|i|,|i|-2, \ldots ; \quad k \geqslant 0, \\
l & =|j|,|j|-2, \ldots,-|j| .
\end{aligned}
$$

The terms $\lambda_{i j k l}$ and $\mu_{i j k l}$ are functions of the parameters $\alpha, \beta, \sigma, a_{5}, a_{7}$, and $a_{9}$ which define the potential. The corresponding equations of motion are

$$
\begin{aligned}
& I_{i}=-\varepsilon^{2} \partial H_{4} / \partial \theta_{i}+O\left(\varepsilon^{4}\right), \\
& \dot{\theta}_{i}=\omega_{i}+\varepsilon^{2} \partial H_{4} / \partial I_{i}+O\left(\varepsilon^{4}\right), \quad i=1,2 .
\end{aligned}
$$

It is evident that the quartic and higher order terms in the potential result in a complicated time dependence of the actions and angles. One expects that in general this time variation will consist of two parts: a "local" oscillation of order $\varepsilon^{2}$, on a time scale comparable to $1 / \omega_{2}$ (i.e., the dynamical time scale), and a more gradual change on a time scale of order $1 / \varepsilon^{2}$ times the dynamical time (e.g., Arnold 1978).

Unfortunately, equations (20) cannot be solved exactly, even if only the terms of $O\left(\varepsilon^{2}\right)$ are retained. However, we are not interested in the small oscillations, but only in the gradual change induced by the quartic terms in the potential. This suggests replacing equations (20) by some sort of averaged set of equations which describe only the gradual change. Hopefully, the resulting system can be solved and will give reasonably accurate approximations to the exact orbits over some interval of time. Krylov and Bogoliubov (see Bogoliubov and Mitropolsky 1961) have proven rigorously that by averaging the right-hand side of equations (20) in a specific way, one indeed obtains a much simpler set of equations, the solutions of which are approximations to the exact solutions.

First write the frequency ratio $\omega_{1} / \omega_{2}$ as

$$
\omega_{1} / \omega_{2}=m / n+\delta \text {, }
$$

where $m$ and $n$ are relatively prime integers and $\delta$, the "detuning parameter," is of $O\left(\varepsilon^{2}\right)$. This representation of the frequency ratio by rational numbers and admitting the irrationals by a small perturbation of the rationals is discussed in detail by Verhulst (1979). Substitute for the action-angle variables in equations (20)

$$
\begin{aligned}
I_{i} & =I_{i}(t), \\
\theta_{1}(t) & =\frac{m}{n} \omega_{2} t+\phi_{1}(t), \\
\theta_{2}(t) & =\omega_{2}+\phi_{2}(t)
\end{aligned}
$$


The error introduced in equation (22b) by neglecting $\delta$ is of $O\left(\varepsilon^{2}\right)$. Since $m / n$ is a rational number, the result of the substitution is that for fixed $(I, \phi)$ the right-hand side of equation (20) is a periodic function of $t$, with period $T$, say. We therefore define a time average $\langle f\rangle$ for any function $f(\bar{I}, \bar{\theta})$ by

$$
\langle f\rangle=\frac{1}{T} \int_{0}^{T} f(\overline{\boldsymbol{I}}, \overline{\boldsymbol{\theta}}) d t,
$$

where it is assumed that substitution (22) is carried out and the integration is performed while keeping $I_{i}$ and $\phi_{i}$ fixed. To first order, the motion is then described by the averaged equations

$$
\begin{aligned}
& \dot{\bar{I}}_{i}=-\varepsilon^{2}\left\langle\partial H_{4}(\bar{I}, \bar{\theta}) / \partial \bar{\theta}_{i}\right\rangle, \\
& \dot{\bar{\theta}}_{i}=\omega_{i}+\varepsilon^{2}\left\langle\partial H_{4}(\bar{I}, \bar{\theta}) / \partial \bar{I}_{i}\right\rangle,
\end{aligned}
$$

where the bar is introduced to indicate that terms of $O\left(\varepsilon^{4}\right)$ have been omitted. By means of the KrylovBogoliubov-Mitropolsky theorem, the solutions $\bar{I}_{i}(t)$ and $\bar{\theta}_{i}(t)$ of these averaged equations are first-order asymptotic approximations to the solutions $I_{i}(t)$ and $\theta_{i}(t)$ of the exact equations of motion (3). Specifically:

$$
\begin{aligned}
& \left|I_{i}(t)-\bar{I}_{i}(t)\right| \leqslant C \varepsilon^{2}, \\
& \left|\theta_{i}(t)-\bar{\theta}_{i}(t)\right| \leqslant C \varepsilon^{2}, \quad \text { for } 0 \leqslant \varepsilon^{2} t \leqslant L T, \quad i=1,2,
\end{aligned}
$$

where $C$ and $L$ are constants of order unity (Bogoliubov and Mitropolsky 1961; Van der Burgh 1974).

The averaging theorem as stated does not make use of the fact that the equations of motion are derivable from a Hamiltonian. However, in the case of a Hamiltonian system we can reduce the necessary amount of computation considerably by noting that the averaged equations (24) are just the equations of motion corresponding to the averaged Hamiltonian $\langle H\rangle$ given by

$$
\langle H\rangle=H^{(0)}(\overline{\boldsymbol{I}})+\varepsilon^{2}\left\langle H_{4}(\overline{\boldsymbol{I}}, \overline{\boldsymbol{\theta}})\right\rangle .
$$

Consider a typical term in the expression for $H_{4}$ in equation (19), after substitution of equation (22):

$$
\begin{aligned}
\lambda_{i j k l} I_{1}^{|i| / 2} I_{2}^{|j| / 2} \cos \left(k \theta_{1}-l \theta_{2}\right) \\
=\lambda_{i j k l} I_{1}^{|i| / 2} I_{2}^{|j| / 2} \cos \left\{[(m / n) k-l] \omega_{2} t\right. \\
\left.\quad+k \phi_{1}-l \phi_{2}\right\} .
\end{aligned}
$$

As long as $m k-n l \neq 0$, this expression will be a periodic function of time, with average over a period $T=$ zero. If, however, $m / n=l / k$, the expression (27) is independent of time and equal to its average. Now. it is clear from the specific form of the Hamiltonian $\mathrm{H}_{4}$ that only four combinations $(k, l)$ occur that do not have zero average for all $(m, n)$. In addition to terms with $k=l=0$, which have nonzero average for all values of $m / n, H_{4}$ contains terms with $|l / k|=1 / 3,1$, or 3 . It follows that the averaged Hamiltonian (26) can take one of only four nontrivial forms, depending on the ratio $m / n$, viz., $m / n \neq\{1 / 3,1,3\} ; m / n=1 / 3 ; m / n=1$; and $m / n=3$. The case $m / n=3$ will not be discussed further, since it is excluded by the condition that $\omega_{1}<\omega_{2}$. It is equivalent to the $1: 3$ case with the coordinate axes interchanged.

The various averaged Hamiltonians are given in Table 1 , both for the nonrotating and rotating cases. The integrals of motion - two in each case-are also given. The coefficients $\mu_{11}, \mu_{12}, \mu_{22}, \mu_{2}, \mu_{4}, \mu_{5}$, and $\mu_{8}$ are functions of the parameters $\alpha, \beta, \sigma, a_{5}, a_{7}$, and $a_{9}$ which define the potential, and are listed in Appendix A. Note that in the nonrotating case we have $\mu_{2}=\mu_{4}=\mu_{8}=0$ and $\mu_{5}=\frac{1}{2} \mu_{12}$.

The occurrence of "discrete" special cases for Hamiltonian systems is a characteristic feature and can be

\begin{tabular}{|c|c|c|c|}
\hline$m / n$ & No Rotation & Rotation & $\begin{array}{l}\text { Integrals } \\
\text { of Motion }\end{array}$ \\
\hline$\neq 1 / 3,1 / 1,3 / 1 \ldots$ & $\begin{aligned} H_{0}= & \kappa_{1} \bar{I}_{1}+\kappa_{2} \bar{I}_{2} \\
& +\varepsilon^{2}\left(\frac{1}{2} \mu_{11} \bar{I}_{1}^{2}+\mu_{12} \bar{I}_{1} \bar{I}_{2}+\frac{1}{2} \mu_{22} \bar{I}_{2}^{2}\right)\end{aligned}$ & $\begin{aligned} H_{0}= & \omega_{1} \bar{I}_{1}+\omega_{2} \bar{I}_{2} \\
& +\varepsilon^{2}\left(\frac{1}{2} \mu_{11} \bar{I}_{1}^{2}+\mu_{12} \bar{I}_{1} \bar{I}_{2}+\frac{1}{2} \mu_{22} \bar{I}_{2}^{2}\right)\end{aligned}$ & $\bar{I}_{1} ; \bar{I}_{2}$ \\
\hline $1 / 1 \ldots \ldots \ldots \ldots \ldots$ & $H_{1: 1}=H_{0}+\frac{1}{2} \varepsilon^{2} \mu_{12} \bar{I}_{1} \bar{I}_{2} \cos \left(2 \bar{\theta}_{1}-2 \bar{\theta}_{2}\right)$ & $\begin{aligned} H_{1: 1}=H_{0}+\varepsilon^{2}\{ & \mu_{4} \bar{I}_{1}^{3 / 2} \bar{I}_{2}^{1 / 2} \sin \left(\bar{\theta}_{1}-\bar{\theta}_{2}\right) \\
& +\mu_{5} \bar{I}_{1} \bar{I}_{2} \cos \left(2 \bar{\theta}_{1}-2 \bar{\theta}_{2}\right) \\
& \left.+\mu_{8} \bar{I}_{1}^{1 / 2} \bar{I}_{2}^{3 / 2} \sin \left(\bar{\theta}_{1}-\bar{\theta}_{2}\right)\right\}\end{aligned}$ & $\bar{I}_{1}+\bar{I}_{2} ; H_{1: 1}$ \\
\hline $1 / 3 \ldots \ldots \ldots \ldots \ldots$ & $H_{1: 3}=H_{0}$ & $H_{1: 3}=H_{0}+\varepsilon^{2} \mu_{2} \bar{I}_{1}^{3 / 2} \bar{I}_{2}^{1 / 2} \sin \left(3 \bar{\theta}_{1}-\bar{\theta}_{2}\right)$ & $\frac{1}{3} \bar{I}_{1}+\bar{I}_{2} ; H_{1: 3}$ \\
\hline
\end{tabular}
represented in a natural way by the averaging technique adopted here (cf. Verhulst 1979). If we had included cubic as well as quartic terms in the potential, we would have found an additional nontrivial form corresponding to $m / n=1 / 2$.

We now consider each case in detail.

TABLE 1

First-Order AVERAged HAMILtonians For $V=V\left(x^{2}, y^{2}\right)$ 
i) $m / n \neq\{1 / 3,1\}$ (No First-Order Resonance)

In this case the equations of motion following from the averaged Hamiltonian $H_{0}$ (cf. Table 1) can be solved immediately. We have

$$
\begin{gathered}
\bar{I}_{i}=E_{i} \quad(i=1,2), \\
\bar{\theta}_{1}=\left[\omega_{1}+\varepsilon^{2}\left(\mu_{11} E_{1}+\mu_{12} E_{2}\right)\right] t+\bar{\theta}_{1}(0), \\
\bar{\theta}_{2}=\left[\omega_{2}+\varepsilon^{2}\left(\mu_{12} E_{1}+\mu_{22} E_{2}\right)\right] t+\bar{\theta}_{2}(0),
\end{gathered}
$$

where $E_{1}, E_{2}, \bar{\theta}_{1}(0)$, and $\bar{\theta}_{2}(0)$ are constants determined by the initial conditions. The normal modes of the linearized system $\left(\bar{I}_{1}=0, \bar{I}_{2}=0\right)$ remain periodic solutions. In this approximation the only difference from the harmonic case is that now the frequencies depend on the actions, i.e., on the orbital amplitudes.

Vandervoort (1979) has computed a first-order integral of the motion for this case (in addition to the total energy). It is easy to show that the average of this integral, as defined in equation (23), is a quadratic expression in $E_{1}$ and $E_{2}$.

\section{ii) $m / n=1$ (1:1 Resonance)}

The properties of the solutions of the equations of motion following from the averaged Hamiltonian $H_{1: 1}$ (cf. Table 1) will be studied in detail in a later paper (de Zeeuw 1983). Since the case $m / n=1$ (henceforth referred to as " $1: 1$ resonance") turns out to be the most important for describing elliptical galaxies, we give here the main results from that analysis.

The actions $\bar{I}_{1}$ and $\bar{I}_{2}$, corresponding to the energies of the fundamental oscillations in the linear approximations, are not conserved separately in this case. However, it is easy to show that

$$
\bar{I}_{1}+\bar{I}_{2}=K_{1},
$$

where $K_{1}$ is a constant determined by the initial conditions. Since $\omega_{2} K_{1}$ is the approximate energy integral, we can write

$$
H_{1: 1}=\omega_{2} K_{1}+\varepsilon^{2} K_{2} .
$$

It follows that, for the averaged equations of motion corresponding to $H_{1: 1}, K_{2}$ is an exact isolating integral of motion independent of $K_{1}$. We find

$$
\begin{aligned}
K_{2}= & \frac{1}{2} \mu_{11} \bar{I}_{1}^{2}+\mu_{12} \bar{I}_{1} \bar{I}_{2}+\frac{1}{2} \mu_{22} \bar{I}_{2}^{2}+\omega_{2}\left(\delta / \varepsilon^{2}\right) \bar{I}_{1} \\
& +\mu_{4} \bar{I}_{1}{ }^{3 / 2} \bar{I}_{2}{ }^{1 / 2} \sin \left(\bar{\theta}_{1}-\bar{\theta}_{2}\right)+\mu_{5} \bar{I}_{1} \bar{I}_{2} \cos \left(2 \bar{\theta}_{1}-2 \bar{\theta}_{2}\right) \\
& +\mu_{8} \bar{I}_{1}{ }^{1 / 2} \bar{I}_{2}^{3 / 2} \sin \left(\bar{\theta}_{1}-\bar{\theta}_{2}\right) .
\end{aligned}
$$

From the averaging theorem (cf. eq. [25]) we know that both $K_{1}$ and $K_{2}$ are approximate integrals of the exact equations of motion (3), with an error of order $\varepsilon^{2}$ on a time scale of order $1 / \varepsilon^{2}$ times the dynamical time. Of course $\omega_{2} K_{1}$ is an $O\left(\varepsilon^{2}\right)$ approximation of the total energy valid for all time. From the above it is clear that $H_{1: 1}$ differs from the total energy by an amount of $O\left(\varepsilon^{4}\right)$. This means that $K_{2}$ is, with an error of $O\left(\varepsilon^{2}\right)$, an approximate integral for all times (Verhulst 1981).

For $\Omega=0$ the expression (31) reduces to the one derived by Verhulst (1979) in his study of epicyclic motion in the meridional plane of an axisymmetric galaxy (the famous "third integral"; cf. Contopoulos 1960) which in turn is a generalization of the expression derived by Contopoulos and Moutsoulas (1965). Saaf (1968) also computed a formal integral for a quartic potential. Here we note one of the advantages of the averaging method as compared to the formal methods used by earlier authors (Contopoulos 1960, 1963; Vandervoort 1979). Whereas both methods give, at least in first order, exactly the same expression for the additional integral, the averaging method at the same time gives an estimate of the accuracy of the approximation and the time scale of validity.

The orbits described by the averaged equations of motion will not be periodic in general. However, as a second isolating integral exists in this case, all of the nonperiodic orbits will be strongly tied to the periodic ones (Ford 1974; Arnold 1978). Their trajectories in phase space lie on well-defined tori surrounding the various stable periodic orbits ("parent" periodic orbits). The tori are parameterized by the value of the second integral. We can consider the orbits in configuration space as a superposition of a guiding center drift provided by the parent periodic orbit and an epicyclic motion around it. The second integral can be interpreted as a measure of the energy in this generalized epicyclic motion. For the orbits given by the exact equations of motion the same is true, but only to first order. Most orbits still are strongly tied to the periodic orbits, but the fraction of these orbits that lie on well-defined tori in phase space generally decreases with increasing energy (e.g., Ford 1974; see also $\S$ III $f$ ).

Clearly it is worthwhile to find the major families of periodic orbits. We summarize the actions and angles, the condition for existence, and the shapes of the various families of periodic orbits that follow from the averaged Hamiltonian $H_{1: 1}$ in Table 2A (no rotation, $\Omega=0$ ) and Table $2 \mathrm{~B}$ (with rotation, $\Omega \neq 0$ ). The conditions for stability are also given. These are obtained by using the second integral $K_{2}$ as a Lyapounov-function (Kurth 1976; Arnold 1978). For the case of no rotation see also Verhulst (1979).

In the absence of rotation the normal modes $\left(I_{i}=0\right)$ are solutions, of course, since they are just the axial orbits, but the periods are now amplitude-dependent. These orbits are called "type I" by Verhulst (1979). For certain energies $K_{1}$ (the respective intervals can be de- 


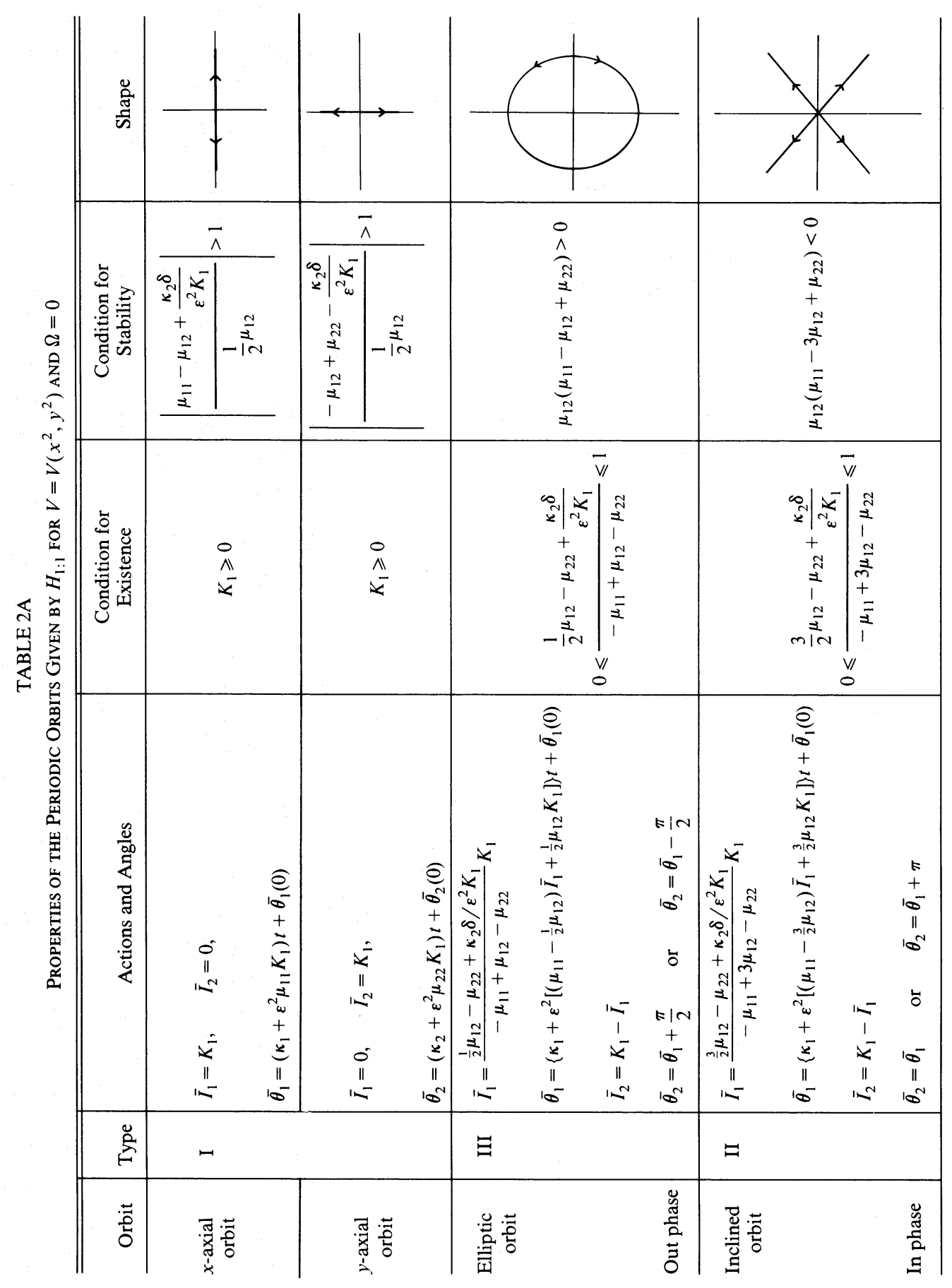




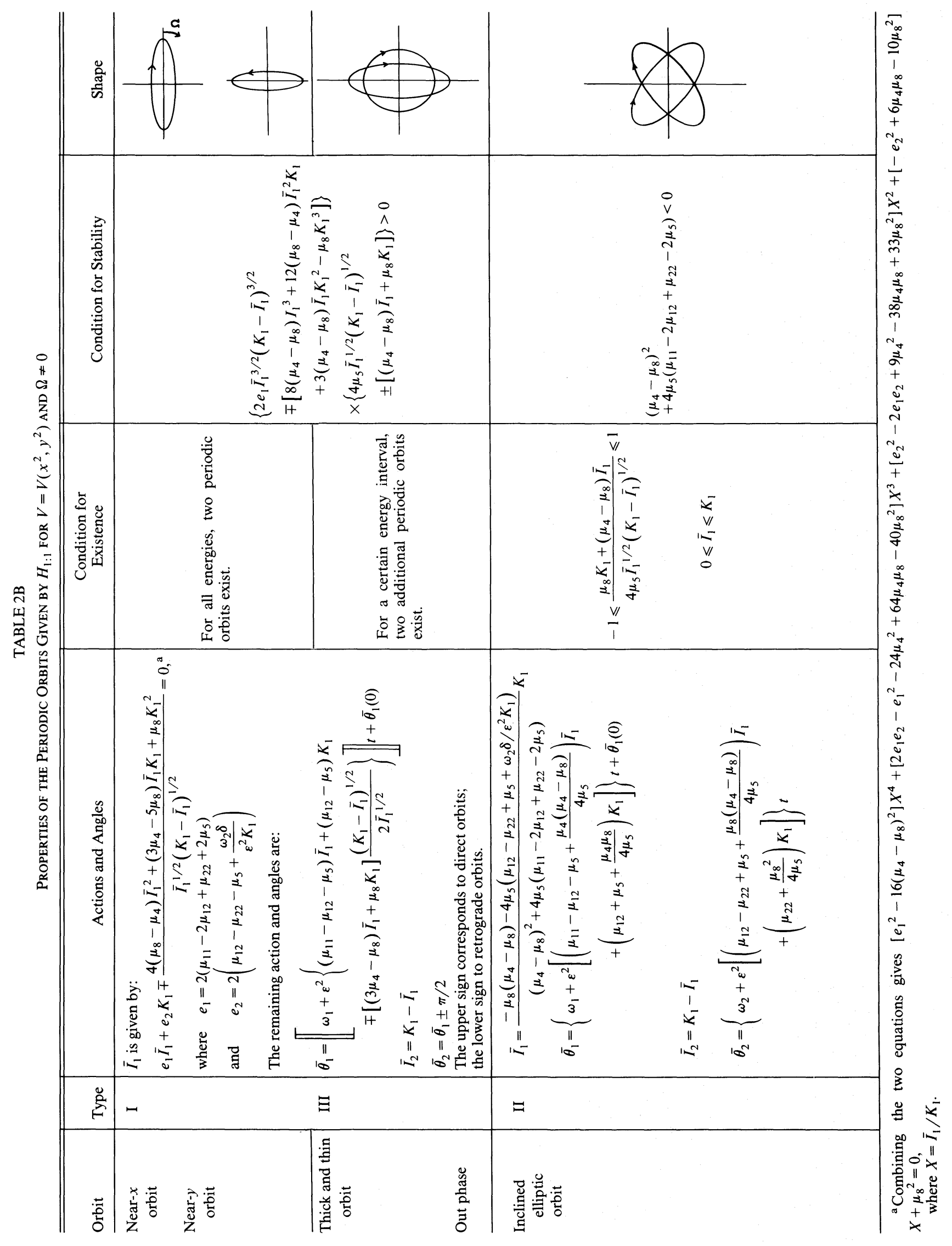


duced from Table $2 \mathrm{~A}$ ) one or two additional orbit families exist: orbits with $\bar{\theta}_{1}-\bar{\theta}_{2}=\{0, \pi\}$ (type II) which are straight lines passing through the origin but inclined to the $x$ - and $y$-axes, and orbits with $\bar{\theta}_{1}-\bar{\theta}_{2}= \pm \pi / 2$ (type III) which are identical ellipses centered on the origin and traversed in opposite directions.

Since orbits corresponding to types II and III do not appear either in the linear or nonresonant case, the structure of phase space in the energy range where these orbits exist will differ qualitatively from that of a harmonic oscillator. We will call this energy interval the "resonance zone."

In the case of a rotating potential, the normal modes are no longer solutions of the equations of motion. However, for all energies there exist a direct $\left(\bar{\theta}_{1}-\bar{\theta}_{2}=\right.$ $-\pi / 2)$ and a retrograde $\left(\bar{\theta}_{1}-\bar{\theta}_{2}=+\pi / 2\right)$ periodic orbit. These are analogous to the type I orbits in the nonrotating potential; at low energies they are very thin, and are elongated in the direction of the $x$ - and $y$-axes, respectively. For certain energies there exist two additional orbits with $\bar{\theta}_{1}-\bar{\theta}_{2}=+\pi / 2$ or $-\pi / 2$. These orbits are typically direct, one stable and the other unstable, and may be considered generalizations of the type III (elliptic) orbits in the nonrotating case. A third family, corresponding to the type II (inclined) orbits, also appears for certain energies; the phase difference $\bar{\theta}_{1}-\bar{\theta}_{2}$ is now unequal to 0 or $\pi$ and depends on the energy. These orbits are ellipses, the symmetry axes of which are inclined to the coordinate axes.

A number of authors have discussed periodic orbits in barred potentials and have obtained results similar to ours. For instance, Contopoulos and Moutsoulas (1965) investigated by analytical and numerical means the $1: 1$ resonance in a model potential containing one cubic term of the form $\varepsilon x y^{2}$, and no detuning. They derived a formal integral of motion for this case and showed that it was well conserved numerically. They also found that three different types of orbits exist, which they called types A, B and C and which are related to three distinct periodic orbit families (corresponding to our types I, II, and III). The same potential with detuning was considered by Contopoulos and Moutsoulas (1966). Andrle (1979 and references therein) considered motion in quartic potentials of the form $V=V\left(x^{2}, y^{2}\right)$ by two different formal methods. Verhulst (1979) used the averaging method to analyse potentials of the form $V=$ $V\left(x, y^{2}\right)$ including all cubic and quartic terms and allowing for detuning, which is required for a study of epicyclic motion in the meridional plane of an axisymmetric galaxy. For the 1:1 resonance he generalized the integral of motion found by Contopoulos and Moutsoulas and showed that it is asymptotic in the sense described above (cf. eqs. [25]). In this more general potential the same three types of periodic orbits still exist.

The present investigation generalizes these results to rotating potentials. We have found that an additional asymptotic integral still exists and have shown that it is valid for all time. The three periodic orbit families also exist, but their shapes are dependent both on the degree of rotation, and on their direction of circulation about the rotation axis.

$$
\text { iii) } m / n=1 / 3(1: 3 \text { Resonance })
$$

The properties of the solutions of the equations of motion corresponding to $H_{1: 3}$ are discussed by de Zeeuw (1983). Again a rigorous asymptotic isolating integral of motion exists in addition to the energy: $K_{2}=\frac{1}{3} \bar{I}_{1}+\bar{I}_{2}$. Note that in the absence of rotation there is no $1: 3$ resonance: $H_{1: 3}$ is identical to the no-resonance Hamiltonian $H_{0}$. This is related to the fact that the potential is even in $x$ and $y$, i.e., $V=V\left(x^{2}, y^{2}\right)$.

In all the above cases there is in first order a second asymptotic integral in addition to the total energy. This is a general property of systems with two degrees of freedom and does not depend on the assumption that $V=V\left(x^{2}, y^{2}\right)$. For higher order resonances we expect that this result is also true (e.g., Sanders and Verhulst 1979). In particular, in general rotating two-dimensional potentials stellar orbits are characterized by an asymptotic integral in addition to the total energy. The fraction of orbits with two integrals is 1 in the harmonic core and decreases as the energy (i.e., the nonlinearity) is increased.

\section{APPLICATION TO A REALISTIC MODEL POTENTIAL}

\section{a) The Potential}

Schwarzschild (1979) has constructed a nonrotating self-consistent triaxial galaxy model by performing a linear superposition of particle orbits in a fixed potential, so that the density distribution assumed in deriving the potential is reproduced. Recently Schwarzschild (1982) has succeeded in constructing a slowly rotating version of the same model. His model galaxies are considerably more realistic than earlier numerical or analytical models, in that their density profile is similar to that observed in real galaxies, and because he made no a priori assumptions about the distribution of stellar velocities (e.g., he did not require the velocity dispersions to be isotropic). Some dynamical properties of the nonrotating model are discussed by Merritt (1980). Wilkinson and James (1982) have shown that these models are likely to be stable for at least a Hubble time (see also Smith and Miller 1982).

The potential used by Schwarzschild is described briefly in Appendix B. We define it to be zero at the origin. As $r=\left(x^{2}+y^{2}\right)^{1 / 2}$ tends to infinity, the potential approaches 1. Using Schwarzschild's (1979) formulae, we find for the five parameters defining the Taylor expansion (eq. [4]) of the potential in the $x-y$ 
plane

$$
\begin{gathered}
\kappa_{1}=0.4241, \quad \kappa_{2}=0.6039 \\
a_{5}=-0.0415, \quad a_{7}=-0.1742, \quad a_{9}=-0.3068 .
\end{gathered}
$$

The quartic potential (4) is a good approximation to the exact potential for $|x|<1.8,|y|<0.9$, corresponding to energies less than about 0.1 . The first-order theory is expected to be valid for the same energy range, since for higher energies the quartic potential allows particles to escape. The unit of length is the core radius (cf. Appendix B). The rescaled version of the potential (eq. [17]) can be obtained by setting $\varepsilon^{2}=0.2$ or 0.3 and changing $a_{5}, a_{7}$, and $a_{9}$ accordingly.

The equidensity surfaces of Schwarzschild's model galaxy have constant axis ratios in the $x-y$ plane of 1:0.625. The equipotential surfaces are rounder, with axis ratios that vary from 1:0.7 near the center to 1:0.8 at an energy of 0.3 .

We shall consider two cases: $\Omega^{2}=0$, and $\Omega^{2}=2 \times$ $10^{-4}$. The latter rotation frequency corresponds to a figure rotation period of about $10^{8} \mathrm{yr}$, if Schwarzschild's model is scaled to a typical galaxy. Real elliptical galaxies generally rotate less quickly than this, so that our two rotation frequencies may be considered extreme cases. The corotation radius of the rotating model lies at $r \approx 25$. Schwarzschild's (1982) model has $\Omega^{2}=1 \times 10^{-6}$; the model constructed by Wilkinson and James (1982) has $\Omega^{2}=1 \times 10^{-5}$.

\section{b) Axial Orbits and Choice of an Energy Scale}

In the absence of rotation, each of the three Hamiltonians derived in $\S \mathrm{II} c$ predicts the same dependence of the frequencies of the axial orbits on their energies. We have (cf. Tables 1 and 2B)

$$
\begin{aligned}
\omega_{i}{ }^{a} & =\omega_{i}+\varepsilon^{2} \mu_{i i} K_{1}, \\
E_{i} & =\omega_{i} K_{1}+\frac{1}{2} \varepsilon^{2} \mu_{i i} K_{1}{ }^{2} .
\end{aligned}
$$

where $\omega_{i}{ }^{a}$ is the frequency of oscillation of the $i$ th axial orbit, $E_{i}$ is the orbital energy, and $\omega_{i}=\kappa_{i}$ for $\Omega=0$. Substituting equation (32b) into equation (32a) gives

$$
\omega_{i}^{a}=\omega_{i}+\varepsilon^{2}\left(\mu_{i i} / \omega_{i}\right) E_{i}+O\left(\varepsilon^{4}\right),
$$

so that the frequencies of the axial orbits depend linearly on their energies, for low energies. Figure 1 shows the dependence of $\omega_{1,2}{ }^{a}$ on $E$ for orbits computed numerically from the model potential. Surprisingly, the dependence is almost linear for energies up to 0.4 , which is far outside the region of validity of the first order approximation.

This numerical property of Schwarzschild's potential suggests the following scheme for extending the analytic

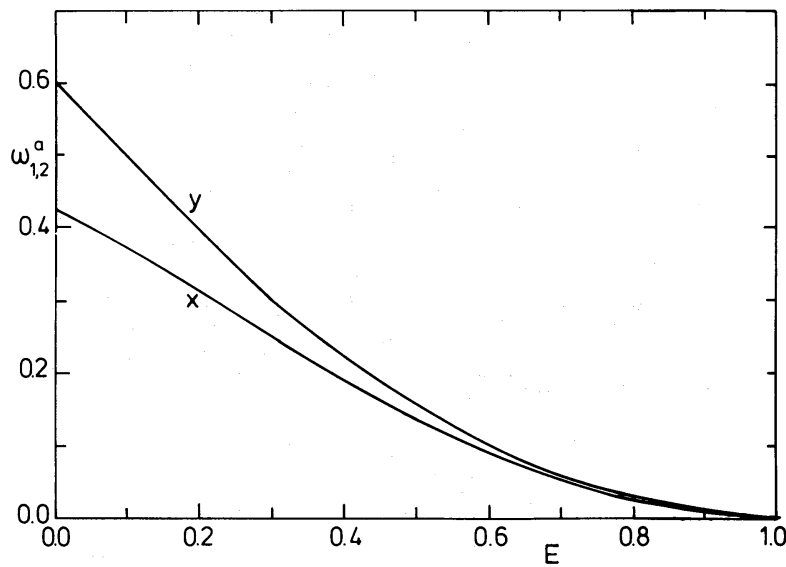

FIG. 1.-Frequency of axial orbits as a function of energy in Schwarzschild's potential. Each of the averaged Hamiltonians of Table 1 predicts a linear dependence of $\omega_{1,2}{ }^{a}$ on $E$.

results, without the necessity of computing higher order approximations to the Hamiltonian. Suppose that we consistently neglect terms of $O\left(\varepsilon^{2}\right)$ in defining the energy of an (analytical) orbit. By "stretching" the energy scale in this way, the frequencies of the normal modes - and hopefully, of the other periodic orbits-can be predicted out to energies of 0.3 or so, where the quartic terms no longer provide a good approximation to the potential. Orbits of these energies typically extend to three or four core radii from the center, so the extension, if it works, is a very valuable one.

\section{c) Choice of a Hamiltonian}

Before making any further comparisons between analytical and numerical results, we must decide which of the three Hamiltonians derived in $\S \mathrm{II} c$ is the appropriate one for describing Schwarzschild's potential. Following the prescription of equation (21), we consider the ratio

$$
\begin{aligned}
\omega_{1} / \omega_{2} & =0.702, & & \Omega^{2}=0 ; \\
& =0.699, & & \Omega^{2}=2 \times 10^{-4} .
\end{aligned}
$$

Expressed as a ratio of integers, $\omega_{1} / \omega_{2}$ is approximately equal to $2 / 3$ or $3 / 4$. Of the two resonant Hamiltonians, $H_{1: 1}$ is clearly a better choice than $H_{1: 3}$, although it is not clear whether the no-resonance Hamiltonian $H_{0}$ might not be best.

However, analysis of the axial orbits has shown that by a suitable definition of the energy, we might hope to extend the analytic results somewhat beyond the region of validity of the asymptotic theory. In this regime, the potential "seen" by a typical orbit is best described in terms of the frequencies of oscillation of the finiteamplitude axial orbits $\omega_{i}^{a}$, and not by the central fre- 
quencies $\omega_{i}$, since a high-energy orbit is relatively unaffected by the shape of the potential in the core. Furthermore, Figure 1 shows that the ratio $\omega_{1}{ }^{a} / \omega_{2}{ }^{a}$ approaches 1 as $E$ increases, since the potential becomes nearly spherical at high energies. We conclude that the potential should be described by the 1:1 Hamiltonian.

There is an additional reason for making this choice. At very low energies, where the analytic results should be most accurate, the choice of a Hamiltonian is relatively unimportant, since the potential is indistinguishable from that of a (rotating) anharmonic oscillator. Consequently, both $H_{0}$ and $H_{1: 1}$ give the same results. At higher energies, however, the resonance Hamiltonian predicts a wealth of detail in the structure of phase space that is not predicted by the nonresonant Hamiltonian. Much of this detail is in fact seen, as will be discussed below.

If the frequency ratio $\omega_{1} / \omega_{2}$ were nearly equal to $1 / 3$, the choice of an averaged Hamiltonian would be more difficult. Presumably, in this case the potential would be best described as a 1:3 resonance at low energies and a 1:1 resonance at high energies.

\section{d) Periodic Orbits}

The simplest periodic orbits are those for which the actions are constant, and the complete set of these was discussed in $\S$ II $c$ for the 1:1 Hamiltonian. We will call such orbits "short" periodic orbits, since they typically cross a given coordinate axis a small number of times before closing, and their period is of the order of a dynamical time. In the present case of a 1:1 resonance, these orbits are, to first approximation, linear combinations of two normal modes with identical frequencies.

The numerical procedure for finding the short periodic orbits in a given potential is straightforward. For orbits that are symmetric with respect to the coordinate axes (all except the type II), specifying the energy leaves only a single free parameter, e.g., the point at which the orbit passes (perpendicularly) through one of the coordinate axes. In the case of the type II orbits in the nonrotating potential, which are straight lines through the origin, this single parameter is the angle which the orbit makes with respect to the axes. Type II orbits in the rotating potential are tilted ellipses and require a two-parameter search, since both their amplitude, and the angle at which they are inclined to the coordinate axes, are unknown.

In the nonrotating potential, the equations of motion are unaffected by the transformation $t \rightarrow-t$, so that every closed orbit has a counterpart with the same figure but opposite angular momentum. Orbits in the rotating potential do not have this property, and retrograde and direct orbits belonging to the same family can have quite different shapes.
TABLE 3

Values of the Parameters Defining $H_{1: 1}$

\begin{tabular}{|c|c|c|}
\hline Parameter & $\Omega=0$ & $\Omega=0.0141$ \\
\hline$\omega_{1} \ldots \ldots$ & 0.4241 & 0.4232 \\
\hline$\omega_{2} \ldots \ldots$ & 0.6039 & 0.6052 \\
\hline & -0.2977 & -0.3008 \\
\hline$\mu_{11} \ldots \ldots \ldots \ldots$ & -0.0854 & -0.0853 \\
\hline$\mu_{12} \ldots \ldots \ldots \ldots$ & -0.2388 & -0.2424 \\
\hline$\mu_{22} \ldots \ldots \ldots \ldots$ & -0.6310 & -0.6251 \\
\hline$\mu_{4}, \ldots \ldots \ldots \ldots$ & 0 & 0.00003 \\
\hline (n. & -0.1194 & -0.1174 \\
\hline$\mu_{8}$ & 0 & -0.0587 \\
\hline
\end{tabular}

i) $\Omega^{2}=0$

The parameters defining the 1:1 Hamiltonian in this case are given in Table 3. The energy, after neglecting terms of order $\varepsilon^{2}$, is

$$
E=\kappa_{2} K_{1}=0.6039 K_{1} \text {. }
$$

As discussed in $\S$ II $c$, the 1:1 Hamiltonian predicts three short periodic orbits in this case: type I (axial orbits); type II (inclined linear orbits), and type III (elliptical orbits).

Axial orbits exist at all energies. For type II orbits the relation between the action $\bar{I}_{1}$ and the energy $\omega_{2} K_{1}$ is

$$
\left(\mu_{11}-3 \mu_{12}+\mu_{22}\right) \bar{I}_{1}+\left(\frac{3}{2} \mu_{12}-\mu_{22}\right) K_{1}=-\kappa_{2} \delta / \varepsilon^{2},
$$

subject to the constraint that $0 \leqslant \bar{I}_{1} \leqslant K_{1}$ (cf. Table $2 \mathrm{~A}$; eq. [29]). It turns out that for the potential chosen here, the coefficient of $\bar{I}_{1}$ on the left-hand side of equation (35) is identically zero, as may be verified from Table 3. It follows that type II orbits do not exist, in first order, in Schwarzschild's potential, except for one energy $E$ given by

$$
E=\kappa_{2} K_{1}=-\kappa_{2}^{2} \delta / \varepsilon^{2}\left(\frac{3}{2} \mu_{12}-\mu_{22}\right) .
$$

At this energy the first-order theory predicts an infinite number of type II orbits since $\bar{I}_{1}$ is undetermined. In other words, at this particular energy every straight line through the center is a periodic orbit. This "degeneracy" may be traced to Schwarzschild's use of a single Legendre polynomial to define the angle dependence of his potential in the $x-y$ plane; as a result, a simple relation exists between the three coefficients of the quartic terms in the potential (4), namely (Appendix B, eq. [B7]).

$$
a_{5}-2 a_{7}+a_{9} \equiv 0 \text {. }
$$

From higher order theory one expects that in the numerical potential only a few of these orbits will occur, if at all (Verhulst 1981). Although the presence of a 


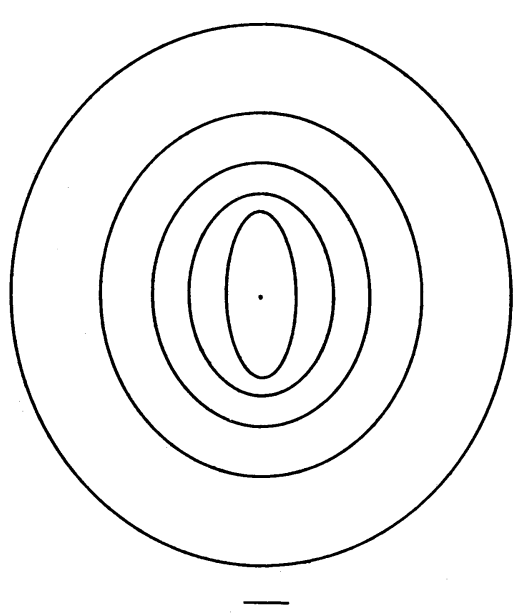

FIG. $2 a$
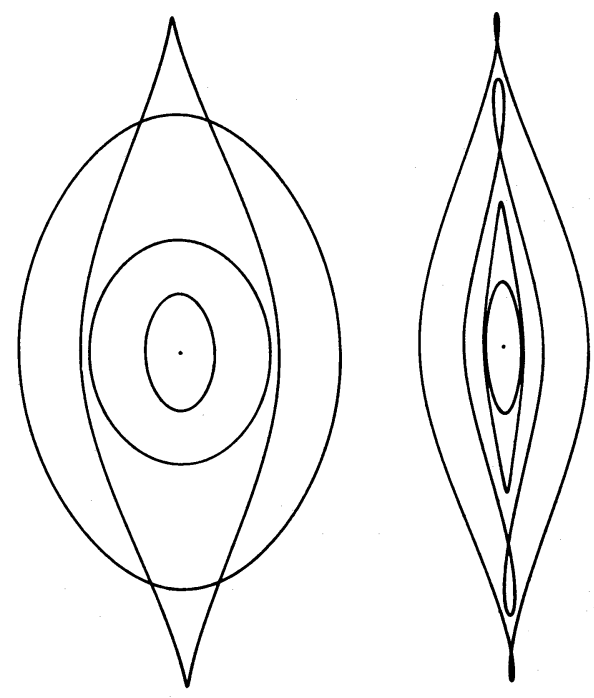

FIG. $2 c$

FIG. 2.-Closed orbits computed with the numerical potential. All orbits are centered on the origin, with the $x$-axis horizontal. The bar at the bottom of each plot has a length of one core radius. (a) Type III orbits in the nonrotating potential, at energies of $(0.3$, $0.4,0.5,0.6,0.7)$. (b) Type I orbits in the rotating potential, at energies of $(0.1,0.2,0.3,0.4,0.5)$ : retrograde (near- $y)$ and direct $($ near- $x$ ). The vertical scale for the near- $x$ orbits has been expanded by a factor of 3. (c) Type III orbits in the rotating potential, at energies of $(0.4,0.6,0.72,0.75)$ : direct "thick" and direct "thin."

finite rotation lifts the degeneracy (cf. Table 2), the value chosen here for $\Omega^{2}$ is small enough [i.e., $\Omega^{2}=$ $O\left(\varepsilon^{2}\right)$ ] that type II orbits are not expected to be important in the rotating potential either. In fact a numerical search shows that the type II orbits almost certainly do not exist in Schwarzschild's potential, nonrotating as well as (slowly) rotating. This point is discussed further in $\S \mathrm{IV}$.
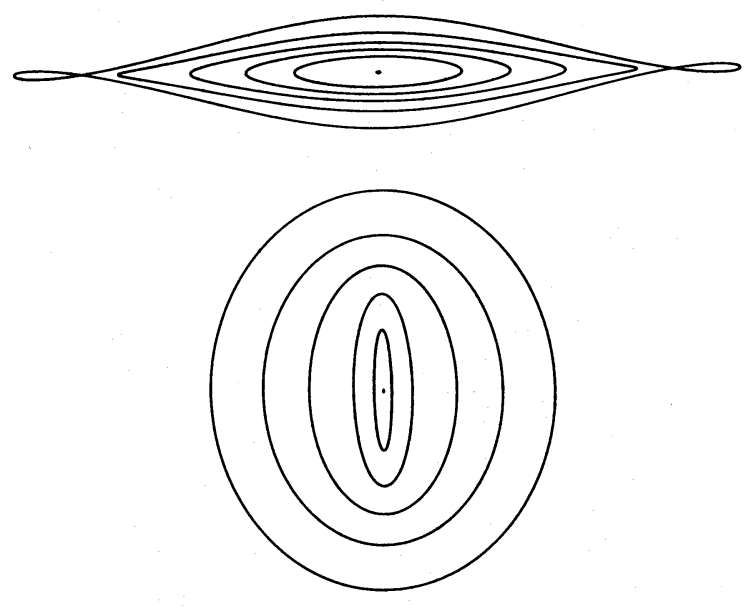

FIG. $2 b$

Type III orbits should exist in the energy range

$0.21 \leqslant E$

A numerical search shows that they "bifurcate" from the $y$-axial orbit at $E=0.27$, in reasonable agreement with the analytical result. At higher energies they quickly become almost circular (cf. Fig. 2a). The energy of bifurcation is equal to the energy at which the $y$-axial orbit becomes unstable to perturbations in the $x$-direction, also in agreement with the analytical prediction (cf. Table 2A).

The relationship between the various periodic orbit families can be displayed by means of a "bifurcation diagram." In Figure $3 a$ we have plotted one of the normalized actions (i.e., amplitudes) predicted by the analytical theory for each of the short periodic orbits, as a function of energy. The straight line $\bar{I}_{1} / K_{1}=1$ corresponds to the $x$-normal mode. The $y$-normal mode is represented by the line $\bar{I}_{1} / K_{1}=0$. The bifurcation at low energies of the type III orbit from the $y$-axial orbit is evident. The instability of the $y$-axial orbit at energies above 0.21 is indicated by a dashed line. The type II orbits exist only at $E=0.39$. They are represented by a vertical line. At this energy the analytical theory predicts a change in stability type of the normal modes: the $x$-axial orbit becomes unstable to perturbations in the $y$-direction and the $y$-axial orbit becomes stable again (cf. Table 2A).

The bifurcation diagram obtained from the numerically calculated orbits is shown in Figure $4 a$, where normalized amplitudes have been plotted in place of actions. The diagram is quite similar to the analytical one for energies up to about 0.3 . The change in stability type of the $x$-and $y$-axial orbits shown in Figure $3 a$ does not occur here, which is of course related to the absence of the type II orbits. 
We conclude that the bulk of Schwarzschild's model potential in the equatorial plane lies in the $1: 1$ resonance zone. With the stretching of the energy scale the analytical technique adequately describes the model to $E \approx 0.3$, which is considerably higher than expected.

$$
\text { ii) } \Omega^{2}=2 \times 10^{-4}
$$

The parameters defining $H_{1: 1}$ are listed in Table 3. For the energy we find

$$
E=\omega_{2} K_{1}=0.6050 K_{1}
$$

The 1:1 Hamiltonian again predicts three orbit families, but now each family contains two distinct members, one retrograde and the other direct. None of these is strictly linear, of course, because of the Coriolis force. The generalized type I and type III orbits appear as solutions to the quartic equation given in the note below Table $2 \mathrm{~B}$ with parameters

$$
\begin{aligned}
& e_{1}=-0.9226, \\
& e_{2}=1.0022-0.2203 / E .
\end{aligned}
$$

Type I orbits exist at all energies and correspond to the normal modes of the linearized equations of motion. According to equation (8), the direct, near- $x$ orbit should have an axis ratio of about 1:0.092 at small energies, while the axis ratio of the retrograde, near- $y$ orbit should be about $0.064: 1$. These orbits are almost linear because $\Omega$ is so small. Type II and type III orbits should exist in the energy ranges

$$
\begin{array}{ll}
0.401 \leqslant E \leqslant 0.403 & \text { (type II) } \\
0.27 \leqslant E & \text { (type III). }
\end{array}
$$

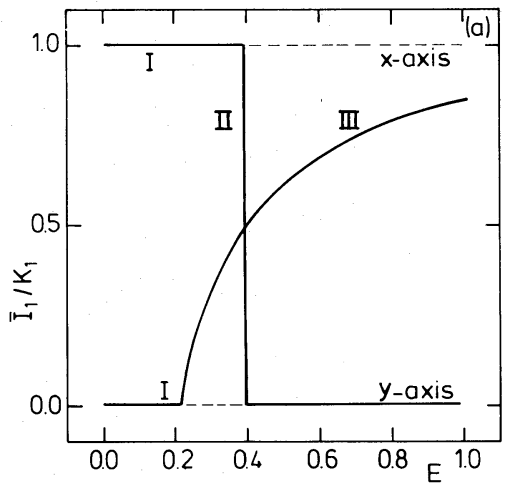

FIG. 3.-First-order analytical bifurcation diagrams for Schwarzschild's model potential. Orbital $x$-amplitude $\bar{I}_{1}$, as a fraction of $K_{1}=\bar{I}_{1}+\bar{I}_{2}$, is plotted against energy for the three families of short periodic orbits. Dotted lines indicate unstable orbits. $(a) \Omega^{2}=0$. The type III orbit branches off from the $y$-axial (type I) orbit when it becomes unstable at $E=0.21$. The type II orbit exists only at $E=0.39$. ( $b$ ) $\Omega^{2}=2 \times 10^{-4}$. The direct (near- $x$ ) type I orbit stays close to the $x$-axis at all energies, while the retrograde (near- $y$ ) becomes more circular. The type II orbits branch off of the near- $x$ orbit at $E=0.401$ and join the $y$-axis at $E=0.403$. The direct type III orbits, one stable and one unstable, appear at $E=0.27$ and exist at all higher energies.

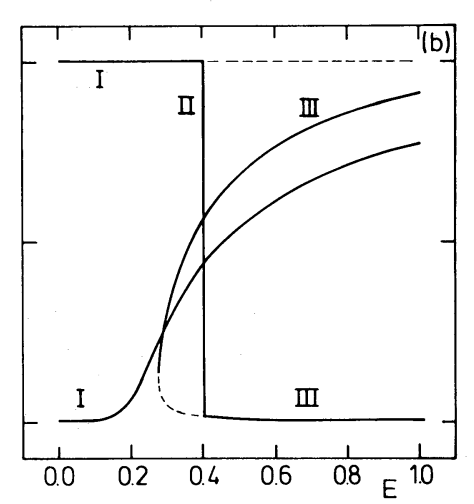

As expected, the finite value of $\Omega$ lifts the degeneracy of Schwarzschild's potential referred to earlier and type II orbits exist. Since $\Omega$ is small the energy range of existence is very narrow, however.

A numerical search in the rotating potential shows that Type II (inclined elliptical) orbits do not exist. This is not surprising since they are predicted to occur at an energy at which the analytical technique is not strictly applicable (cf. § III $a$ ). The type I orbits are shown in Figure $2 b$. At small energies they are nearly elliptical, but outside the harmonic region of the potential they quickly change shape: the near- $x$ orbit remains close to the $x$-axis but becomes three-lobed, and the near- $y$ orbit becomes nearly circular.

The type III orbits are found to exist for $0.37 \leqslant E \leqslant$ 0.75 . At $E=0.37$ the two orbits are identical, but at higher energies they differ, one becoming thicker than the other (Fig. 2c). The thick orbit is stable, and the thin orbit is unstable. Qualitatively this is in excellent agreement with the analytical theory. However, the energy of first appearance of these orbits is underestimated analytically. At $E=0.75$ the thick and the thin orbit join and disappear. This is probably associated with the Lindblad resonance, which occurs at a similar energy.

As in the nonrotating case, connections between the orbit families are most easily seen in a bifurcation diagram. Figure $3 b$ shows the analytical version. The type I orbits clearly reduce to the normal modes at low energies. With increasing energy the near- $y$ orbit becomes nearly round; the near- $x$ orbit remains very close to the axis. The type II orbits branch off from the near- $x$ orbit at $E=0.401$ and join the thin orbit at $E=0.403$. The appearance of the unstable thin and stable thick type III orbits at $E=0.27$ is also evident. Note how changes in stability type are connected with the (dis)appearance of the various orbit families. 

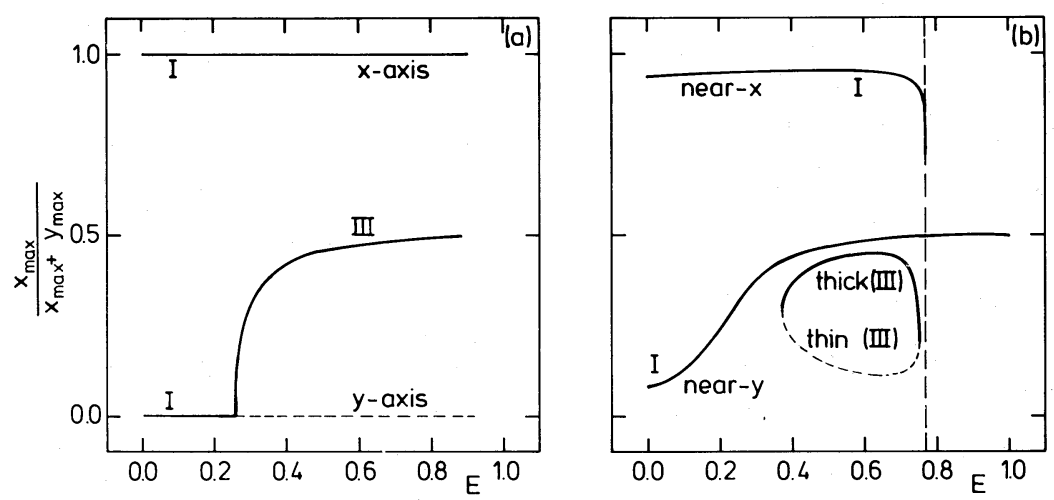

FIG. 4. - Numerical bifurcation diagrams. Orbital $x$-amplitude $x_{\max }$, as a fraction of $x_{\max }+y_{\max }$, is plotted against energy. $(a) \Omega^{2}=0$. The type III orbit branches off from the $y$-axial (type I) orbit when it becomes unstable at $E=0.27$. The type II orbit does not exist. (b) $\Omega^{2}=2 \times 10^{-4}$. The direct and retrograde type I orbits behave almost as in the analytic theory. Type II orbits do not exist. Type III orbits appear at $E=0.37$ and disappear at $E=0.75$, forming a closed loop in the bifurcation diagram. The dashed line indicates the energy of corotation.

The numerical bifurcation picture (Fig. $4 b$ ) compares well to the analytical one for $E \leqslant 0.3$. At higher energies there are differences: the type II orbit does not exist, as explained above, and as a result a change in stability type of the near axial orbits does not occur either. The type III orbits form a closed curve in the diagram and do not join with any of the other families.

Figure $4 b$ is very similar to Figure 6 of Contopoulos and Papayannopoulos (1980), who studied motion in nearly axisymmetric rotating potentials. Their orbits $x_{2}$ and $x_{3}$ may be compared to our thick and thin type III orbits, since they are also direct and disappear at large radii. Contopoulos and Papayannopoulos interpreted the disappearance of these orbits in terms of the Lindblad resonance, which occurs at a similar radius in their models. Although the Lindblad resonance is not strictly defined in a strongly nonaxisymmetric potential (cf. Van Albada and Sanders 1982), we suspect that the disappearance of the type III orbits has a similar explanation in our case. Note that the resonance leading to these orbits can either be described as a 1:1 resonance, as we do, or as a 2:1 resonance between the frequencies of the "unperturbed" circular orbit and the perturbing epicycle, if the epicycle is described in the usual rotating frame defined by Lindblad and Oort.

\section{e) Surfaces of Section}

It is possible to integrate the averaged equations of motion in the general case, giving the explicit dependence of $(\bar{I}, \bar{\theta})$ on time for an arbitrary orbit. The resulting expressions are very unwieldy, however. A much more useful way of investigating families of orbits is through surfaces of section (Poincaré 1899; see also Henon and Heiles 1964; Contopoulos 1973). In the present case we consider surfaces defined by $y=0$ and fixed values of the energy, and take as our coordinates $(x, \dot{x})$. Consequents (points of intersection between the orbit and the surface of section) are constrained to lie within the region bounded by

$$
\frac{1}{2} \dot{x}^{2}+V(x, 0)=E
$$

If a second isolating integral exists, the consequents of a single orbit will lie along a curve, called the "invariant curve." In the first order theory, invariant curves are defined by

$$
K_{2}[x, \dot{x}, 0, \dot{y}(E)]=\text { constant }
$$

where $K_{2}$ is the nonclassical integral of equation (31). Orbits computed numerically can be plotted on a surface of section by recording the values $(x, \dot{x})$ whenever $y=0$. The degree of correspondence between the numerically calculated surfaces of section and those calculated from equation (37) provides a "global" test of the accuracy of the analytical technique.

The behavior of the surfaces of section as a function of energy is shown in Figures 5 (no rotation) and 6 (rotation), computed both analytically and numerically. The most important feature is the onset of the resonance zone and its associated orbits at $E=0.2-0.3$; at lower energies the invariant curves are similar to those of an anharmonic oscillator. The general shape of the invariant curves is very well described by the analytical second integral at low energies; however, the predicted energy of appearance of the resonance zone is somewhat off, as noted above in connection with the periodic orbits.

The correspondence is less good at higher energies. While the theory predicts a return to stability of the $y$-axial orbit, and a corresponding change in the character of the surrounding orbits, no such change is ap-

FIG. 5. - Surfaces of section in the $(x, \dot{x})$ plane for $\Omega^{2}=0, y=0$, and $E=(0.2,0.3,0.4,0.5) .(a)-(d)$, top to bottom analytical; $(e)-(h)$, top to bottom numerical. 

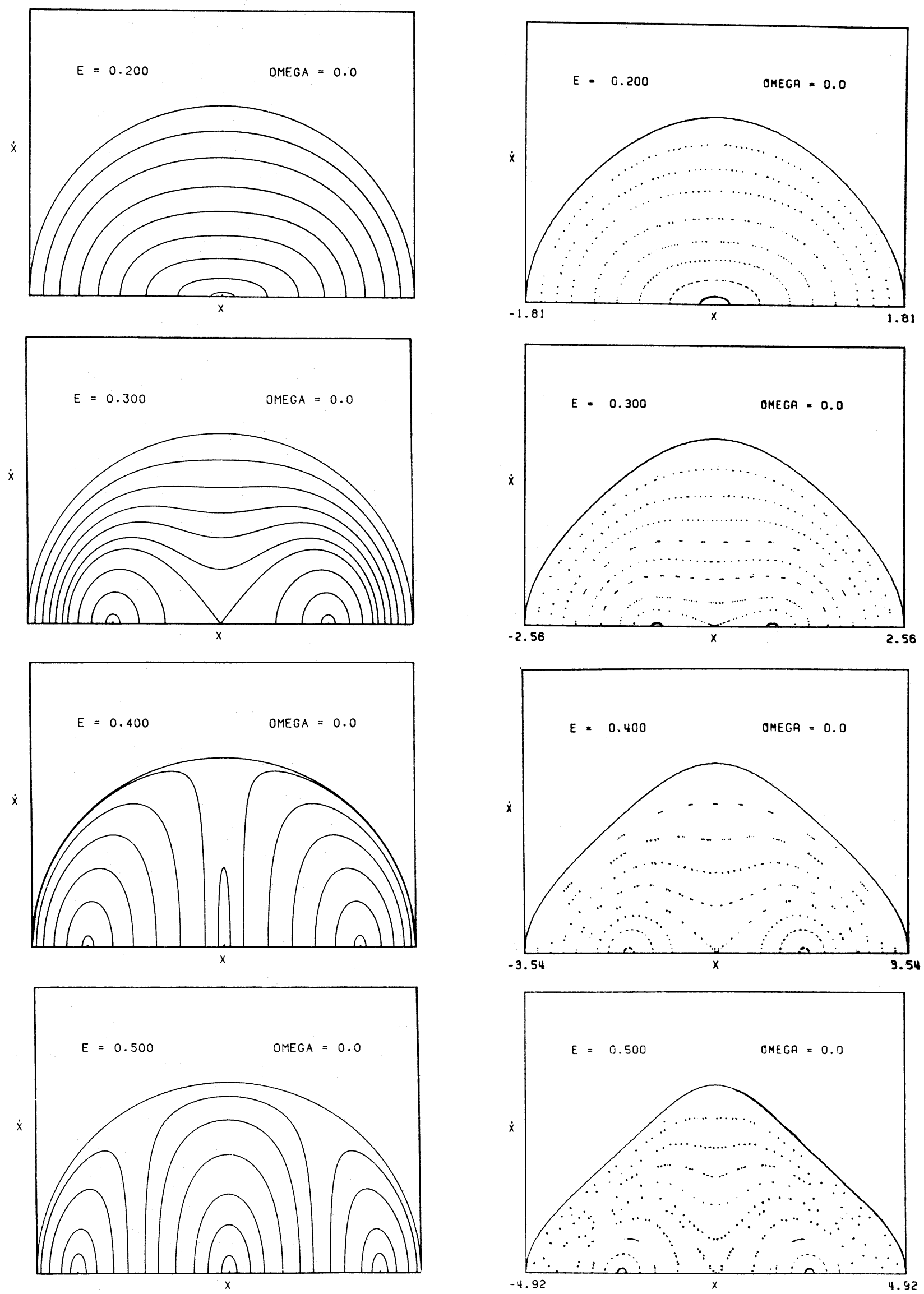

FIG. 5 

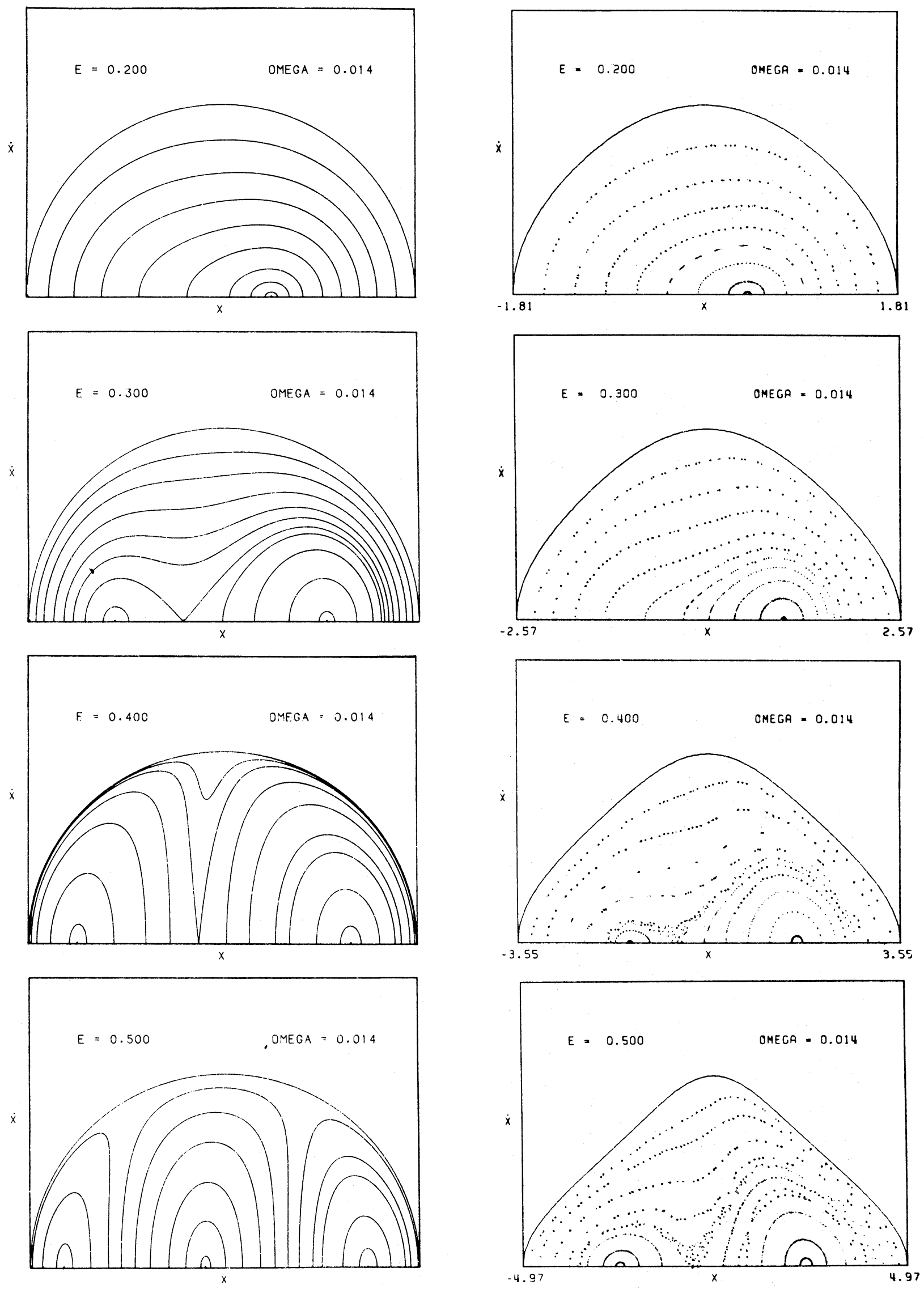

FIG. 6 


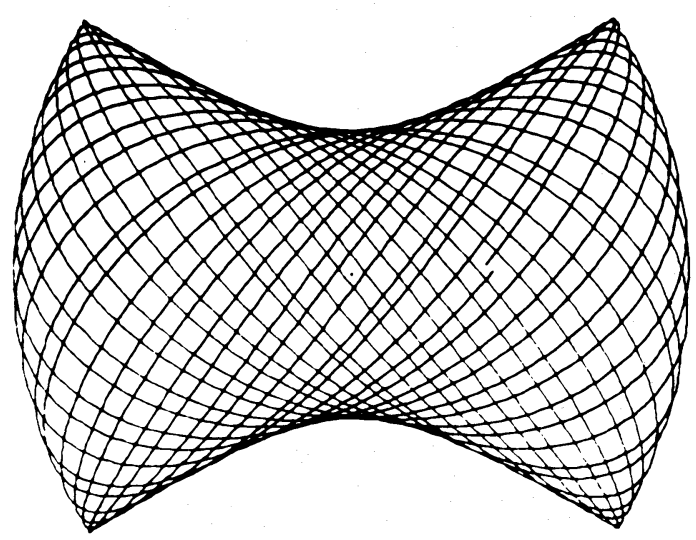

FIG. $7 a$

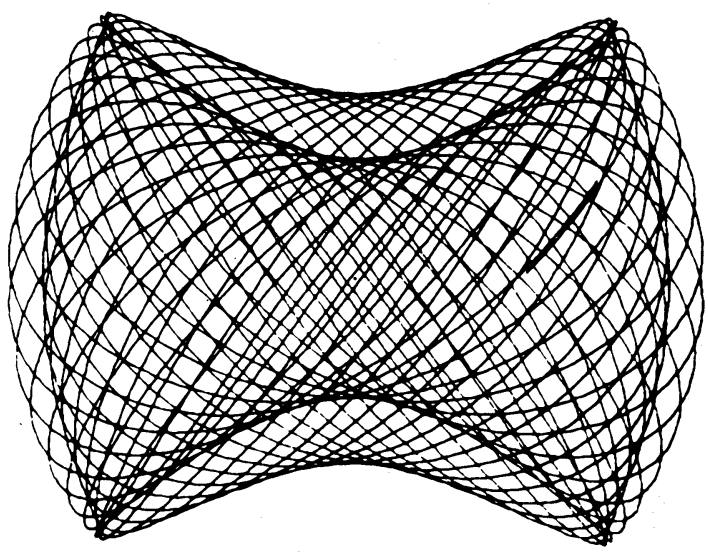

FIG. $7 c$

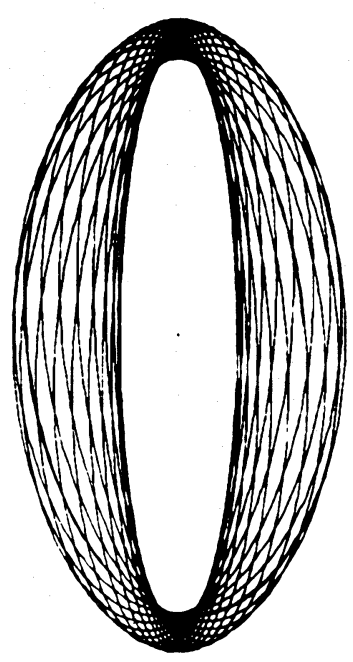

FIG. $7 b$

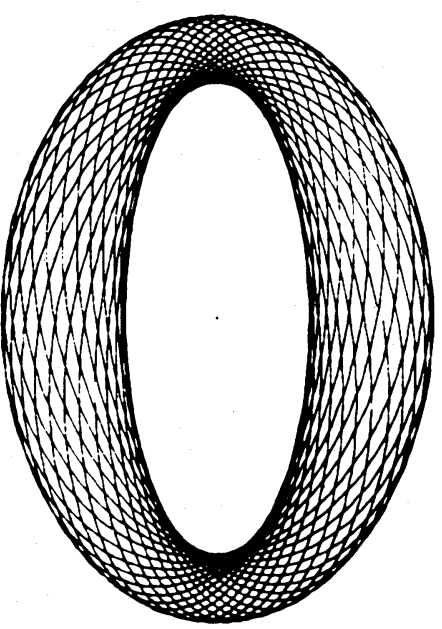

FIG. $7 d$

FIG. 7.- Typical nonperiodic box and tube orbits in Schwarzschild's potential, for $E=0.3$. ( $a$ ) Box orbit, $\Omega^{2}=0$. (b) Tube orbit, $\Omega^{2}=0$. This orbit lies around the type III closed orbit. (c) Box orbit, $\Omega^{2}=2 \times 10^{-4}$. (d) Tube orbit, $\Omega^{2}=2 \times 10^{-4}$. This orbit lies around the type I closed orbit. All orbits are on the same scale. The bar has a length of one core radius.

parent in the numerical surfaces of section. Instead, the description of phase space given by the first-order theory for energies just inside the resonance zone is found to be qualitatively correct at higher energies. In other words, the potential can be described as a 1:1 resonance everywhere outside of the core, and, in the case of rotation, inside the Lindblad resonance (cf. the preceding section). This important result is discussed further in $\S \mathrm{V}$.

Vandervoort (1979) has constructed analytical surfaces of section for a rotating quartic potential in the absence of resonances. Not surprisingly, his figures are very similar to ours at low energies (i.e., outside of the resonance zone). Because he took a somewhat larger value for $\Omega$, the effect of rotation on the invariant curves can be seen more clearly in his diagrams.

Four typical (i.e., nonperiodic) orbits in the numerical potential are illustrated in Figure 7. Box orbits (Ollongren 1962) have as their parent the $x$-axial orbit and exist at all energies. The four corners of the box are points of zero velocity in the $x-y$ plane at which the orbital angular momentum changes sign. A star in a box orbit therefore reverses its sense of progression around the rotation axis every time it reaches one of the four

Fig. 6. - Surfaces of section in the $(x, \dot{x})$ plane for $\Omega^{2}=2 \times 10^{-4}, y=0$, and $E=(0.2,0.3,0.4,0.5)$. $(a)-(d)$, analytical; $(e)-(h)$, numerical. 
corners of the box. In the nonrotating case the star thus simply retraces its path, but in the rotating case direct and retrograde motion are distinct so that the direct part of the box is different from the retrograde part. This effect can clearly be seen in Figure $7 c$. The time-averaged density of a box orbit is strongly peaked in the four corners. Tube orbits are tied to the type III orbits; they have a well-defined angular momentum and avoid the origin. Their appearance is not appreciably influenced by rotation. The importance of these two orbit families in constructing selfconsistent galaxy models has been elucidated by Schwarzschild (1979, 1981a) (cf. also $\S \mathrm{V})$.

\section{f) Stochastic Orbits}

Some of the consequents in both the rotating and nonrotating numerical potentials do not form simple curves in the surfaces of section but fill up finite areas. In both cases, comparison of the numerical and analytical surfaces of section show that the "parent" orbit of the badly behaved consequent is an unstable one, either the $y$-axial orbit or the "thin" type III orbit. Evidently, the onset of instability for these orbits corresponds to a change in character of the second isolating integral over a finite volume in phase space. However, these orbits are not free to wander ergodically over the entire energy surface since much of phase space is filled by orbits which do have a second isolating integral. Following Goodman and Schwarzschild (1981), we will call these orbits "stochastic."

The existence of stochastic regions around unstable orbits is well documented in the literature (e.g., Henon and Heiles 1964; Ford 1974), and can be traced to what is sometimes called an "interaction of resonances" (Jaeger and Lichtenberg 1972; Contopoulos 1973, 1978b; Arnold 1978). An orbit starting anywhere in a stochastic region will eventually fill it up completely, although this may take a very long time (Goodman and Schwarzschild 1981). First-order perturbation theory can predict the possible existence of a stochastic region by giving the energy at which a simple periodic orbit becomes unstable. Furthermore, it can give an upper limit to the extent of such a stochastic region. Since we find that in first order all orbits possess a second approximate integral, which is a constant of the motion within an error of $O\left(\varepsilon^{2}\right)$ for all times, a stochastic region cannot occupy a fraction of the whole energy surface larger than $O\left(\varepsilon^{2}\right)$. Of course, this estimate holds only in the region where first order perturbation theory is valid.

In some cases studied (cf. Henon and Heiles 1964), the size of the stochastic region increases rapidly with energy until it fills up the entire energy surface, and motion is ergodic. To assess the importance of stochasticity in the Schwarzschild model potential, the fraction of the two-dimensional phase space contained in the stochastic region was determined as a function of energy

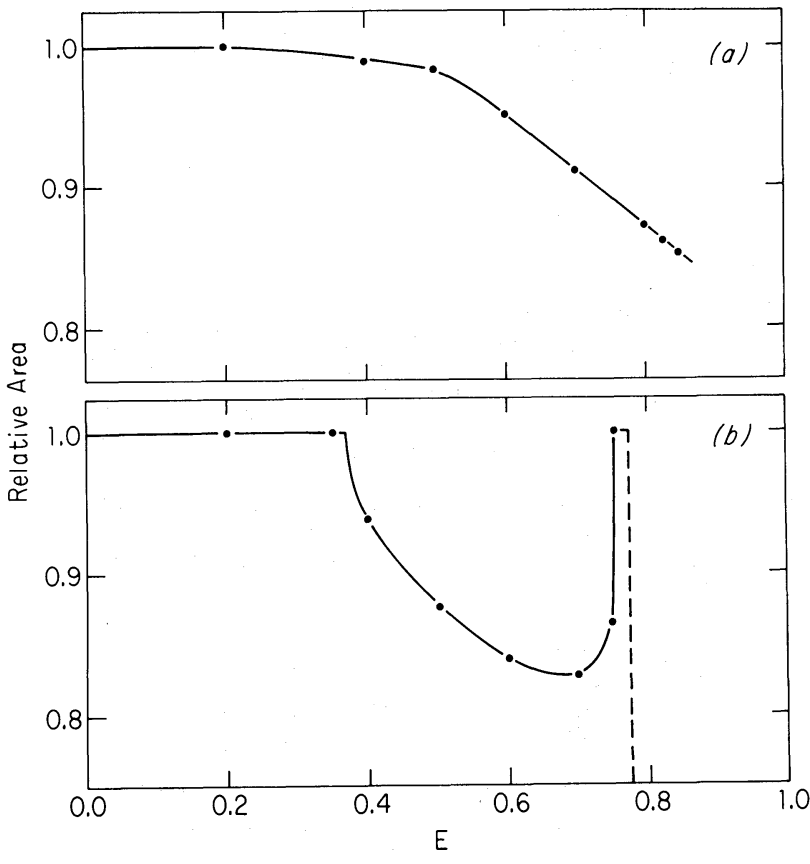

FIG. 8.-Relative area of the numerical surfaces of section covered by well-defined invariant curves. Dashed line indicates energy of corotation. (a) $\Omega^{2}=0$. The stochastic region is tied to the unstable $y$-axial orbit. ( $b$ ) $\Omega^{2}=2 \times 10^{-4}$. The stochastic region is tied to the unstable "thin" type III orbit and disappears at $E=0.75$.

for rotating and nonrotating surfaces of section. Stochastic orbits were run for a very long time $\left(\sim 10^{10} \mathrm{yr}\right.$ in typical model units), and the area which they filled in the surfaces of section measured with a planimeter. The results are shown in Figure 8.

In the nonrotating potential the stochastic region first appears at the onset of instability of the $y$-axial orbit, at an energy of 0.27 , and thereafter increases in size as function of energy, but rather slowly. There is no indication of a rapid transition to almost complete ergodicity, as was found by Henon and Heiles. In the rotating potential the stochastic region is tied to the thin type III orbit, which only exists in the energy range $0.37<E<$ 0.75 . It therefore reaches a maximum size, $17 \%$ of the total area in this case, before disappearing. The relatively small importance of the stochastic orbits at high energies is due to the fact that Schwarzschild's potential approaches an integrable (i.e., spherical) one for large $E$, whereas the Henon-Heiles potential becomes progressively less integrable at high energies.

\section{GENERALIZATION}

In the preceding section we have seen that the orbits in the equatorial plane of Schwarzschild's model galaxy-nonrotating as well as rotating-are characteristic of a $1: 1$ resonance. Here we discuss the generality of this result. Using the analytical formulae given in § II 
and Table 2, we consider the effect of variations in the shape of the density distribution and the rotation frequency. We also discuss the importance of type II orbits in a general triaxial potential. Although the results are formally valid only in the core, they give a qualitative indication of the orbital behavior at larger radii.

\section{a) Variations of the Axis Ratios}

Let $p$ be the ratio of major to minor axis $(x: z)$ of the equidensity surfaces, and $q$ the ratio of median to minor axis $(y: z)$; by definition, $p \geqslant q \geqslant 1$. For an investigation of the effect of variations of $p$ and $q$ on the results obtained above, we need the gravitational potential, or at least its Taylor expansion about the origin, as a function of $p$ and $q$. The potential used by Schwarzschild in the construction of his model galaxy is available only in the form of a numerical table. One could in principle repeat Schwarzschild's potential derivation for different values of $p$ and $q$, but that is a rather cumbersome approach. Fortunately, Schwarzschild (1981 b) has devised an extremely simple analytical approximation to the potential, which we give in Appendix B. Although the approximation was intended only as a representation of the specific potential used in constructing the model galaxy (having $p=2, q=5 / 4$ ), it is applicable for different values of $p$ and $q$, as long as care is taken that the corresponding density distribution does not become negative.

Figure 9 shows the extent of the resonance zonewhich was defined in $\S$ II $c$ (ii) as the energy interval in which the resonant periodic orbits (types II and/or III) exist - as a function of $p / q$ (elongation in the $x-y$ plane) for various values of $q$ (elongation in the $y-z$ plane), for $\Omega=0$. We consider values of $p / q$ up to about 2 . Few ellipticals are more elongated than this (e.g., Binggeli 1980), although some galactic bars have more extreme axis ratios (Kormendy 1981). The case $q=1$ corresponds to prolate galaxies; the effect of varying $q$ on the resonance zone is generally much smaller than of varying $p / q$.

For $p / q=1$ the potential is axisymmetric and the type III orbits exist at every energy; they are circular and stable. As $p / q$ increases the potential becomes increasingly nonaxisymmetric, and the type III orbits first appear at progressively higher energies. This is of course a result of the "detuning" of the potential, which forces the orbit to make use of the quartic terms to match frequencies in the $x$ - and $y$-directions. The upper energy limits to the resonance zone shown in Figure 9, as found from the expressions in Table 2A, should be considered as formal only since our first-order approximation is certainly not valid at such high energies; in practice the potential will become rounder at large radii, and the resonance zone will extend to infinity, as it does in Schwarzschild's model potential.

As discussed above, the type II orbits do not exist in first order in Schwarzschild's nonrotating potential, and this result holds also for the analytical approximation of Appendix B, regardless of axis ratio. The following argument suggests that orbits similar to the type II are never important in slowly rotating potentials. Precisely linear orbits can only exist in regions where the potential gradient points along a straight line. In axisymmetric potentials this condition is satisfied everywhere; in ellipsoidal potentials it is only satisfied along the axes. If, however, the elongation axis of the potential were different at large and small radii, it is reasonable to suppose that a nearly linear closed orbit might exist, since a test particle released from a large enough radius would be deflected from a radial path first in one sense, and then in the other, as it fell inward.

Schwarzschild's potential does not have this property. However, the Taylor expansion up to quartic terms does, at least in certain regions, as can easily be seen by plotting the equipotential curves corresponding to equation (17). This is because the quartic terms tend to deform the harmonic potential radically at energies just
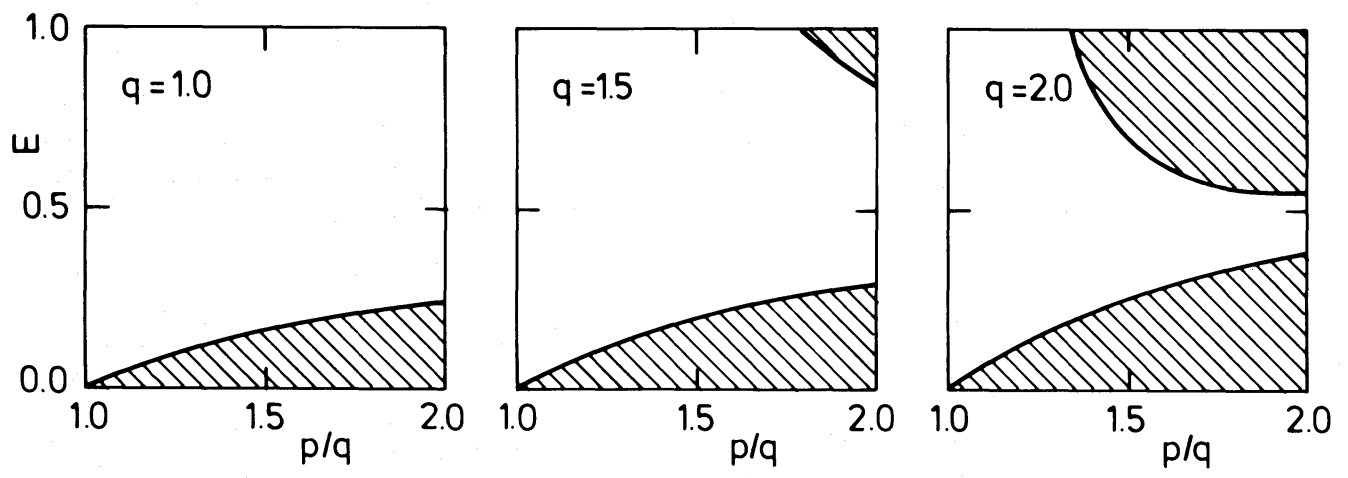

FIG. 9. - Formal extent of the resonance zone as a function of model elongation $p / q$, for various values of $q$, the degree of elongation in the $y-z$ plane. The regions outside the resonance zone are shaded. The resonance zone becomes less important as the detuning increases. 
below where the expansion breaks down. Numerical integration of orbits in the quartic potential shows that nearly linear closed orbits, inclined to the symmetry axes, do in fact exist for $\Omega=0$, and their behavior as a function of energy is similar to that described in $\S$ III $d$ (i). We have also verified that inclined orbits exist in a potential which is similar to the analytical potential of Appendix B but contains an extra term which causes the elongation axis to be different at large and small radii. Elliptical galaxies have been observed which show a shift in their isophotal major axes (e.g., NGC 596; cf. Williams 1981), and it is possible that their density distributions might be capable of supporting inclined closed orbits analogous to type II. However, we suspect that type II orbits do not play any very important role in the dynamics of elliptical galaxies.

\section{b) Variations of Rotation Speed}

We have seen in § III that a small figure rotation has no very important effect on the properties of orbits. In Figure 10 we show the calculated extent of the resonance zone as a function of $\Omega$ for Schwarzschild's model galaxy, i.e., $p=2, q=5 / 4$. The energy of first appearance of both type II and type III orbits increases with $\Omega$, rather more rapidly for type III than type II (recall that a finite $\Omega$ allows type II orbits to exist in first order in Schwarzschild's potential). The different behavior of the two families can be understood by noting that the type II orbits, which always come near the center, are less affected by changes in the potential shape than the nearly circular type III orbits. As explained in the above, for energies larger than $\sim 0.3$ the results are formal only, but Figure 10 is probably correct in predicting the disappearance of type III orbits for large $\Omega$.

\section{c) Effect of Asymmetric Perturbations}

Implicit in the discussion up to now has been the assumption that the potential is symmetric with respect to the three principal planes, i.e., $V=V\left(x^{2}, y^{2}\right)$. Real elliptical galaxies often show small to moderate asymmetries, due presumably to the effect of companions, incomplete relaxation, etc. The time-dependent simulation of Wilkinson and James (1982) suggests that even isolated triaxial galaxies can maintain S-shaped oscillations over a Hubble time. It is therefore important to investigate what changes result from the addition of the asymmetric terms $a_{6} x^{3} y$ and $a_{8} x y^{3}$ to the Taylor expansion of the potential (4). This will be discussed in detail by de Zeeuw (1983). One consequence is that for $a_{6} \neq 0$ the $1: 3$ resonance occurs in first order, even for $\Omega=0$. In other words, the properties of the orbits in a galaxy with $\omega_{1}{ }^{a} / \omega_{2}{ }^{a} \approx 1 / 3$ will be very different if $\Omega=0$ and $a_{6}=0$. Fortunately, such an elongated potential is probably not relevant to elliptical galaxies, as discussed above.

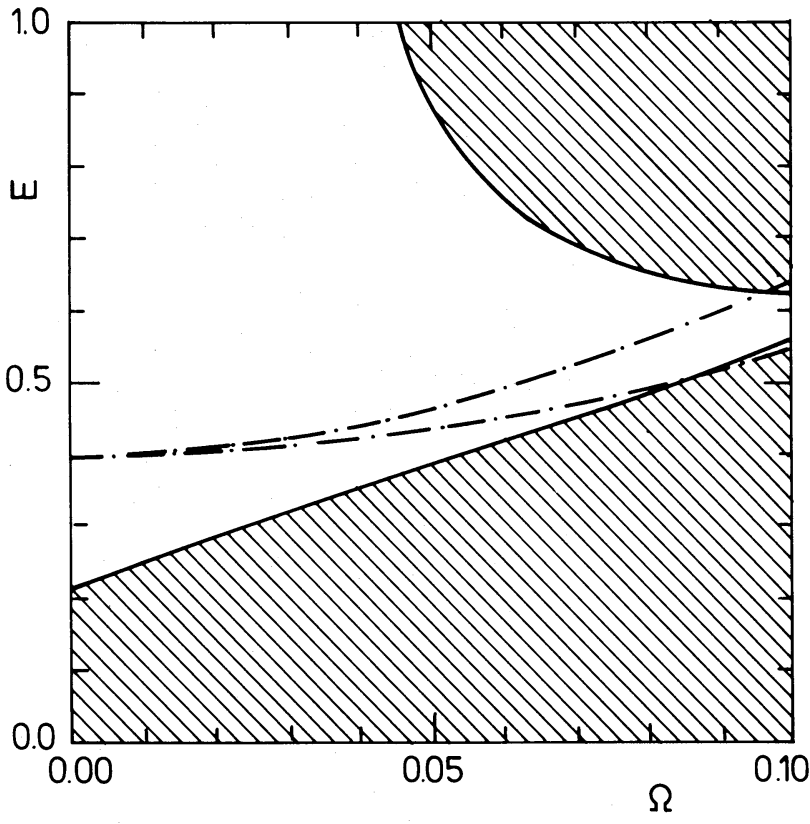

FIG. 10.-Formal extent of the resonance zone as a function of rotation frequency $\Omega$, for $(p, q)=(2,5 / 4)$. Solid lines mark the boundary of the range of existence of the type III orbits. The dashed lines bound the existence region of the type II orbits. The regions outside the resonance zone are shaded. The resonance zone becomes less important at higher rotation speeds.

The 1:2 resonance is associated with cubic (e.g., $x^{2} y$ ) terms in the potential and does not occur unless these are present, regardless of the presence of the $x^{3} y$ or $x y^{3}$ terms. This case is discussed in detail by de Zeeuw (1983).

For the purposes of the present paper the most important question is of course whether the properties of the orbits found for the 1:1 resonance are influenced appreciably by the assumption of symmetry. De Zeeuw (1983) finds that this is not the case; the number of short periodic orbit types that may exist is always three, although the orbital shapes change somewhat. We conclude that the major orbit families found here are likely to be the only ones present in a real elliptical galaxy.

\section{DISCUSSION}

The method of averaging described here has a number of advantages over the standard perturbation techniques, used for example by Contopoulos (1960), Saaf (1968), and Vandervoort (1979) for constructing approximate integrals of the motion in galactic potentials. These are (cf. Verhulst 1979) as follows: 1. Averaging is conceptually and computationally much simpler than solving the Liouville equation for the integrals of motion, even though computer algorithms now exist for generating formal integrals to any desired order (Contopoulos 1966; Gustavson 1966; Giorgilli and Galgani 1978). 
2. Resonances are handled in a natural way by the method of averaging. Expression of a Hamiltonian in terms of action-angle variables as in equations (18) and (19) immediately shows which resonances may occur to the order one is considering.

3. Averaging for the frequency ratio of interest yields an approximate Hamiltonian which can be used to find explicit expressions for the simplest closed orbits. The results are not formal but rigorous: the accuracy and the time interval of the approximations are known explicitly (cf. eq. [25]).

The existence of low-order resonances is crucial in constructing self-consistent galaxy models from a given potential. This may be seen as follows. Hunter (1974) has shown that homogeneous triaxial ellipsoids cannot exist in dynamical equilibrium, since no combination of orbits, calculated in the corresponding (harmonic) potential, is capable of reproducing the assumed density distribution. This is because a harmonic potential, even though it has three independent integrals, can only support box orbits (cf. Fig. 7), which spend a large fraction of their time near the core; any superposition of them gives a density profile that is strongly peaked toward the center, and this is inconsistent with the constant density profile assumed in calculating the orbits. One way around this problem is to allow two of the axes of the ellipsoid to be equal, so that one component of the angular momentum is conserved. Analytic solutions do exist in this case (e.g., Bisnovatyi-Kogan and Zel'dovich 1970), but there is no obvious way to generalize them to nonaxisymmetric potentials where the angular momentum is not conserved.

Schwarzschild (1979) was the first to show how the characteristic forms of orbits in systems with three effective integrals of motion can be used to construct selfconsistent triaxial models. Just as in the case of the homogeneous ellipsoid, he found that three effective integrals, sufficient to define box orbits, existed at every energy in his model galaxy. However, the box orbits always gave too large a density near the long $(x-)$ axis. His solution was to invoke a second family of orbits, the three-dimensional $x$-tubes, which are cylindrically symmetric about the $x$-axis and contribute no density there. The parent family of $x$-tubes is obviously a $1: 1$ closed orbit lying in the $y-z$ plane, analogous to the type III orbits found here in the equatorial plane. It follows that Schwarzschild's numerical solution could probably not have been constructed without the existence of a $1: 1$ resonance in the $y-z$ plane. This resonance obviously plays a role similar to the angular momentum integral in axisymmetric potentials, in that it allows orbits to remain always at large distances from the center, in tubes around the stable closed orbit. In fact, the extra integral found here may be shown to reduce to the angular momentum when the potential is axisymmetric, as it must (see also de Zeeuw 1982).
The situation is a little more complicated if figure rotation is permitted; triaxial solutions have been found in this case without invoking a resonance (e.g., Freeman $1966 b$; Vandervoort 1980). However, in all these models it is primarily the rotation which supports the figure in its nonspherical shape, and not the integrals; in fact, these models always require a unique relation between the figure rotation speed and the degree of elongation. For general rotation speeds - and, in particular, for the low rotation speeds of elliptical galaxies-resonances must always play a role.

We conclude, therefore, that the existence of a resonance zone, in addition to three effective integrals, is probably a necessary condition for the dynamical equilibrium of a triaxial galaxy.

That Schwarzschild's potential exhibits a 1:1 resonance in the equatorial plane is not surprising. Even for very elongated density distributions the potential surfaces tend to be nearly spherical, and as long as the density is a decreasing function of radius, the ratio $\omega_{1}^{a} / \omega_{2}^{a}$ will always tend toward unity as the orbital energy is increased. The results obtained here are primarily useful in showing how the extent of the resonance zone varies with variations in the potential. Figures 9 and 10 suggest that systems which are either very noncircular, or have a high rotation speed, cannot support a large resonance zone, for the reasons given in $\S \mathrm{IV}$. This is, of course, also true for systems which are nearly homogeneous, as shown by Hunter (1974). It follows that self-consistent triaxial galaxy models are probably difficult or impossible to construct in these cases.

The above argument for the prevalence of the $1: 1$ resonance in the equatorial plane of course holds in any of the three principal planes of a triaxial galaxy. Therefore it is very likely that the general orbits in most elliptical galaxies are characteristic of a $1: 1: 1$ resonance between the three fundamental orbital frequencies, i.e., that their phase space structure is best described by a $1: 1: 1$ Hamiltonian. Clearly, it is very important to perform a detailed analysis of the major orbit families in the general, slowly rotating three-dimensional case. The "spectral" method of Binney and Spergel (1982) should be a useful tool for this purpose. The method of averaging described here is just as easily applied to the threedimensional case, and some results have been given by de Zeeuw (1982). A comparison with numerical calculations, like those of Heisler, Merritt, and Schwarzschild (1982) and Magnenat (1982), will be made in a future paper. It seems likely that averaging applied to a threedimensional Hamiltonian will give a good understanding of the orbital behavior throughout most of a triaxial elliptical galaxy.

The authors are primarily indebted to Martin Schwarzschild for his sustained interest and encourage- 
ment throughout the long course of this work. E. Dekker's guidance in the initial stages is also acknowledged. H. C. van de Hulst, F. Verhulst, C. A. Norman, M. Schwarzschild, and G. Contopoulos made detailed criticisms of the manuscript. We benefited also from discussions with J. J. Binney and E. F. van Dishoeck. Much of this work was carried out while both authors were at Princeton University. D. M. was supported in part by a National Science Foundation graduate fellowship, and T. de Z. thanks het Leids Kerkhoven Bosscha Fonds for providing travel assistance. This work was supported in part by NSF grants AST 78-23796 and AST 81-21485.

\section{APPENDIX A}

\section{AVERAGED HAMILTONIANS}

The averaged Hamiltonians are given in Table 1. Here we list the expressions for the coefficients in the three separate cases.

$$
\text { I. } m / n \neq\{1 / 3,1\}
$$

We have

$$
\begin{aligned}
& \mu_{11}=\frac{1}{4}(\sigma / \beta)^{2}\left(3 a_{5}+2 \beta^{2} a_{7}+3 \beta^{4} a_{9}\right), \\
& \mu_{12}=\frac{1}{2}(\sigma / \beta)(\sigma / \alpha)\left[3 \alpha^{2} a_{5}+\left(1+\alpha^{2} \beta^{2}\right) a_{7}+3 \beta^{2} a_{9}\right], \\
& \mu_{22}=\frac{1}{4}(\sigma / \alpha)^{2}\left[3 \alpha^{4} a_{5}+2 \alpha^{2} a_{7}+3 a_{9}\right],
\end{aligned}
$$

and the constants $\alpha, \beta$, and $\sigma$ were defined in equations (8) and (11). For $\Omega=0$ this reduces to

$$
\begin{gathered}
\mu_{11}=\frac{3}{4} a_{5} / \kappa_{1}^{2}, \quad \mu_{12}=\frac{1}{2} a_{7} / \kappa_{1} \kappa_{2}, \quad \mu_{22}=\frac{3}{4} a_{9} / \kappa_{2}^{2} . \\
\text { II. } m / n=1 / 3 \\
\omega_{1}=\frac{1}{3} \omega_{2}+O\left(\dot{\varepsilon}^{2}\right)
\end{gathered}
$$

and

$$
\mu_{2}=\frac{1}{2}(\sigma / \beta)^{3 / 2}(\sigma / \alpha)^{1 / 2}\left[-\alpha a_{5}+\beta(1+\alpha \beta) a_{7}-\beta^{3} a_{9}\right]
$$

For $\Omega=0$ this reduces to

$$
\mu_{2}=0
$$

thus, the first order $1: 3$ resonance exists only for $\Omega \neq 0$.

$$
\begin{gathered}
\text { III. } m / n=1 \\
\omega_{1}=\omega_{2}+O\left(\varepsilon^{2}\right)
\end{gathered}
$$

and

$$
\begin{aligned}
& \mu_{4}=\frac{1}{2}(\sigma / \beta)^{3 / 2}(\sigma / \alpha)^{1 / 2}\left[-3 \alpha a_{5}+\beta(1-\alpha \beta) a_{7}+3 \beta^{3} a_{9}\right], \\
& \mu_{5}=\frac{1}{4}(\sigma / \beta)(\sigma / \alpha)\left[-3 \alpha^{2} a_{5}+\left(1+4 \alpha \beta+\alpha^{2} \beta^{2}\right) a_{7}-3 \beta^{2} a_{9}\right], \\
& \mu_{8}=\frac{1}{2}(\sigma / \beta)^{1 / 2}(\sigma / \alpha)^{3 / 2}\left[-3 \alpha^{3} a_{5}-\alpha(1-\alpha \beta) a_{7}+3 \beta a_{9}\right]
\end{aligned}
$$


For $\Omega=0$ we have

$$
\mu_{5}=a_{7} / 4 \kappa_{2}^{2}, \quad \mu_{4}=\mu_{8}=0,
$$

so that when $\Omega=0$ and $V=V\left(x^{2}, y^{2}\right)$, only $\mu_{5} \neq 0$. Note that in this case $\mu_{5}=\frac{1}{2} \mu_{12}$.

\section{APPENDIX B}

SCHWARZSCHILD'S MODEL POTENTIAL

The density may be written

$$
\rho(x, y, z)=f(r)-P_{2}^{0}(\theta, \phi) g(r)+P_{2}^{2}(\theta, \phi) h(r),
$$

where

$$
\begin{aligned}
& P_{2}^{0}(\theta, \phi)=\left(3 \cos ^{2} \theta-1\right) / 2=\left(2 z^{2}-x^{2}-y^{2}\right) / 2 r^{2}, \\
& P_{2}^{2}(\theta, \phi)=3 \sin ^{2} \theta \cos 2 \phi=3\left(x^{2}-y^{2}\right) / r^{2} .
\end{aligned}
$$

The function $f(r)$, which gives the dominant $r$-dependence at small radii, has the form of a modified Hubble profile:

$$
f(r)=\left(1+r^{2}\right)^{-3 / 2}
$$

The spherical harmonics in the second and third terms are chosen so as to create equidensity surfaces which are symmetric about the three principal planes, but with unequal axis ratios; in this case, the major and minor axes are in the $x$ - and $z$-directions, respectively. The functions $g(r)$ and $h(r)$ are calculated numerically in such a way as to make the axis ratios of the equidensity surfaces nearly independent of radius. Schwarzschild assumed axis ratios of 1:1.25:2. The resulting distribution is not ellipsoidal, being somewhat "dimpled" as seen from the short and intermediate axes. The central density is equal to 1 . The core radius (the radius at which the surface density equals $1 / 2$ ) is also normalized to 1 . The gravitational potential corresponding to equation (B1) can be written

$$
\phi(x, y, z)=u(r)-P_{2}^{0}(\theta, \phi) v(r)+P_{2}^{2}(\theta, \phi) w(r),
$$

where $u, v$, and $w$ are easily derived from Poisson's equation and the expressions for the density. Setting $z=0$ and adding the centrifugal force term gives the potential which was used above in numerically calculating orbits.

Because the potential of equation (B3) is not easily modified to describe galaxies of different axis ratios, Schwarzschild $(1981 b)$ has derived a simple analytical expression with arbitrary axis ratios that reproduces his model potential to reasonable accuracy. The potential corresponding to the first term in equation (B1) may be written exactly:

$$
u(r)=-(1 / r) \log \left[r+\left(1+r^{2}\right)^{1 / 2}\right]+1 .
$$

For $v$ and $w$, assume the functional forms

$$
\begin{aligned}
& v(r)=-c_{1} r^{2} /\left(1+c_{2} r^{2}\right)^{3 / 2} \\
& w(r)=-c_{3} r^{2} /\left(1+c_{4} r^{2}\right)^{3 / 2}
\end{aligned}
$$

Require that the axis ratios of the density distribution corresponding to equation (B4) be the same in the limits of small and large $r$. A straightforward calculation using the expressions in Schwarzschild (1979) yields

$$
c_{1}=d_{1}{ }^{3 / 5} d_{2}{ }^{2 / 5}, \quad c_{2}=\left(d_{1} / d_{2}\right)^{2 / 5}, \quad c_{3}=d_{3}{ }^{3 / 5} d_{4}{ }^{2 / 5}, \quad c_{4}=\left(d_{3} / d_{4}\right)^{2 / 5},
$$


where

$$
\begin{aligned}
& d_{1}=\left(2 p^{2} q^{2}-p^{2}-q^{2}\right) / 14\left(p^{2} q^{2}+p^{2}+q^{2}\right) \\
& d_{2}=(1 / 6)\left[1-3 /\left(p^{3}+q^{3}+1\right)\right] \\
& d_{3}=\left(p^{2}-q^{2}\right) / 28\left(p^{2} q^{2}+p^{2}+q^{2}\right), \\
& d_{4}=\left(p^{3}-q^{3}\right) / 12\left(1+p^{3}+q^{3}\right)
\end{aligned}
$$

and $p$ and $q$ are the axis ratios, referred to the short axis, at small and large $r$. The potential is zero in the center and approaches 1 as $r$ goes to infinity. The density functions corresponding to equation (B4) are given by

$$
\begin{aligned}
& g(r)=3 c_{1} c_{2}\left(7+2 c_{2} r^{2}\right) r^{2} /\left(1+c_{2} r^{2}\right)^{7 / 2}, \\
& h(r)=3 c_{3} c_{4}\left(7+2 c_{4} r^{2}\right) r^{2} /\left(1+c_{4} r^{2}\right)^{7 / 2}
\end{aligned}
$$

For the case $(p=2, q=1.25)$, we have

$$
c_{1}=0.06408, \quad c_{2}=0.65456, \quad c_{3}=0.01533, \quad c_{4}=0.98067
$$

A comparison with the density calculated numerically from equation (B1) and Poisson's equation used by Schwarzschild (1979) shows that the axis ratios deviate by no more than $15 \%$ from their central values in this case.

The Taylor expansion of the potential up to quartic terms is

$$
\phi(x, y, z)=\frac{1}{2} \kappa_{1}{ }^{2} x^{2}+\frac{1}{2} \kappa_{2}{ }^{2} y^{2}+\frac{1}{2} \kappa_{3}{ }^{2} z^{2}+\frac{1}{4} a_{5} x^{4}+\frac{1}{2} a_{7} x^{2} y^{2}+\frac{1}{4} a_{9} y^{4}+\frac{1}{2} b_{11} x^{2} z^{2}+\frac{1}{2} b_{13} y^{2} z^{2}+\frac{1}{4} b_{16} z^{4},
$$

where

$$
\begin{array}{lll}
\kappa_{1}^{2}=\frac{1}{3}-c_{1}-6 c_{3}, & \kappa_{2}^{2}=\frac{1}{3}-c_{1}+6 c_{3}, & \kappa_{3}^{2}=\frac{1}{3}+2 c_{1}, \\
a_{5}=-\frac{3}{10}+3 c_{1} c_{2}+18 c_{3} c_{4}, & a_{7}=-\frac{3}{10}+3 c_{1} c_{2}, & a_{9}=-\frac{3}{10}+3 c_{1} c_{2}-18 c_{3} c_{4}, \\
b_{11}=-\frac{3}{10}-\frac{3}{2} c_{1} c_{2}+9 c_{3} c_{4}, & b_{13}=-\frac{3}{10}-\frac{3}{2} c_{1} c_{2}-9 c_{3} c_{4}, & b_{16}=-\frac{3}{10}-6 c_{1} c_{2} .
\end{array}
$$

Note that

$$
a_{5}-2 a_{7}+a_{9} \equiv 0
$$

regardless of axis ratios.

\section{REFERENCES}

Aarseth, S. J., and Binney, J. J. 1978, M.N.R.A.S., 185, 227.

Andrle, P. 1979, Bull. Astr. Inst. Czechoslovakia, 30, 362.

Arnold, V. 1978, Mathematical Methods of Classical Mechanics

(New York: Springer).

Binggeli, B. 1980, Astr. Ap., 82, 289.

Binney, J. J. $1978 a, M . N . R . A . S ., 183,501$ 1978b, Comments Ap., 8, 2.

Binney, J. J., and Spergel, D. 1982, Ap. J., 252, 308

Bisnovatyi-Kogan, G. S., and Zel'dovich, Ya. B. 1970, Astrofisika, 6, 387 (translated as 1973 Astrophysics, 6, 207).

Bogoliubov, N. N., and Mitropolsky, J. A. 1961, Asymptotic Methods in the Theory of Nonlinear Oscillations (New York: Gordon and Breach).

Contopoulos, G. 1960, Zs. Ap., 49, 273

Contopoulos, G. 1963, A.J., 68, 763. . 1966, Ap. J. Suppl., 13, 503.
Contopoulos, G. 1973, in Dynamical Structure and Evolution of Stellar Systems, Proc. Third Advanced Course of the Swiss Society of Astronomy and Astrophysics, ed. L. Martinet and M. Mayor (Sauverny: Geneva Observatory).

$$
\text { 1975, Ap. J., 201, } 566 .
$$

$1978 a$, Astr. Ap., 64, 323.
$1978 b$, in Theoretical Principles in Astrophysics and Relativity, ed. N. R. Lebovitz, W. H. Reid, and P. O. Vandervoort (Chicago: University of Chicago Press), p. 93.

Contopoulos, G., and Moutsoulas, M. 1965, A.J., 70, 817. 1966, A.J., 71, 687.

Contopoulos, G., and Mertzanides, C. 1977, Astr. Ap., 61, 477.

Contopoulos, G., and Papayannopoulos, Th. 1980, Astr. Ap., 92, 33.

Davies, R. I. 1981, M.N.R.A.S., 194, 879. 
Davies, R. I., Efstathiou, G., Fall, S. M., Illingworth, G., and Schechter, P. L. 1982 Proc. CECAM Workshop on the Formation, Structure and Evolution of Galaxies, ed. J. Audouze and C. A. Norman (Orsay: CECAM), p. 188.

de Zeeuw, P. T. 1982, Proc. CECAM Workshop on the Formation, Structure and Evolution of Galaxies, ed. J. Audouze and C. A. Norman, p. 11.

$\frac{\text {. 1983, in preparation. }}{\text { de Zeeuw, P. T., Franx, M., Meys, J., Brink, K., and Habing, H. J. }}$ 1983, in IAU Symposium 100, Internal Kinematics and Dynamics of Galaxies, ed. E. O. Athanassoula (Dordrecht: Reidel), in press.

Ford, J. 1974, in Lectures in Statistical Physics, ed. W. C. Schieve and J. S. Turner (New York: Springer), p. 228

Freeman, K. C. $1966 a, M . N . R . A . S ., 133,47$. .1966b, M.N.R.A.S., 134, 1.

Giorgilli, A., and Galgani, L. 1978, Celestial Mechanics, 17, 267.

Goodman, J., and Schwarzschild, M. 1981, Ap. J., 245, 1087.

Gustavson, G. 1966, A.J., 71, 670 .

Hagihara, Y. 1974, Celestial Mechanics, Vol. 2 (Cambridge: M.I.T. Press).

Heiligman, G., and Schwarzschild, M. 1979, Ap. J., 233, 872.

Heisler, J., Merritt, D., and Schwarzschild, M. 1982, Ap. J., 258 , 490.

Henon, M., and Heiles, C. 1964, A.J., 69, 73.

Hunter, C. 1974, M.N.R.A.S., 166, 633.

Illingworth, G. 1977, Ap. J. (Letters), 218, L43.

1981, in The Structure and Evolution of Normal Galaxies,

ed. S. M. Fall and D. Lynden-Bell (London: Cambridge University Press), p. 27.

Jacobi, C. G. J. 1836, Compte Rendus Acad. Sci. Paris, 3, 59.

Jaeger, E. F., and Lichtenberg, A. J. 1972, Ann. Phys., 71, 319.

Jeans, J. H. 1916, M.N.R.A.S., 76, 70.

King, I. R. 1978, Ap. J., 222, 1.

Kormendy, J. 1981, in The Structure and Evolution of Normal Galaxies, ed. S. M. Fall and D. Lynden-Bell (London: Cambridge University Press), p. 85.

Kormendy, J., and Illingworth, G. 1982, Ap. J., 256, 460.
Kurth, R. 1976, Elements of Analytical Dynamics (Oxford: Pergamon Press)

Leach, R. 1981, Ap. J., 248, 485

Lynden-Bell, D. 1962, M.N.R.A.S., 124, 95.

Magnenat, P. 1982, Astr. Ap., 108, 89.

Martinet, L., and Mayer, F. 1975, Astr. Ap. 44, 45.

Merritt, D. 1980, Ap. J. Suppl., 43, 435

Miller, R. H. 1978, Ap. J., 223, 122.

Ollongren, A. 1962, Bull. Astr. Inst. Netherlands, 16, 241.

Poincaré, H. 1899, Les Methodes Nouvelles de la Mecanique Celeste, Vol. 3 (Paris: Gauthier-Villars).

Peterson, C. J. 1978, Ap. J., 222, 84.

Richstone, D. D. 1980, Ap. J., 238, 103.

Saaf, A. F. 1968, $A p$. J., 154, 483.

Sanders, J. A., and Verhulst, F. 1979, in Lecture Notes in Mathematics, Vol. 711 ed. F. Verhulst (Berlin: Springer), p. 209. 1981, Preprint.

Schechter, P. L., and Gunn, J. F. 1979, Ap. J., 229, 472

Schwarzschild, M. 1979, Ap. J., 232, 236.

Schwarzschild, M. 1981 a, in The Structure and Evolution of Normal Galaxies, ed. S. M. Fall and D. Lynden-Bell (London: Cambridge University Press), p. 43. $1981 b$, private communication. 1982, Ap. J., 263, 599.

Smith, B. F., and Miller, R. H. 1982, Ap. J., 257, 103.

Stark, A. A. 1977, Ap. J., 213, 368.

Van Albada, T. S., and Sanders, R. H. 1982, M.N.R.A.S., 201, 303.

Van der Burgh, A. H. P. 1974, thesis, University of Delft. 1976, J. Sound and Vibration, 49, 93.

Vandervoort, P. O. 1979, Ap. J., 232, 91. 1980, Ap. J., 240, 478

Verhulst, F. 1979, Phil. Trans. Roy. Soc. London, 290, 435. 1981, private communication.

Wilkinson, A., and James, R. A. 1982, M.N.R.A.S., 199, 171.

Williams, T. B., 1981, Ap. J., 244, 458.

Williams, T. B., and Schwarzschild, M. 1979a, Ap. J., 227, 56. 1979b, Ap. J. Suppl., 41, 209.

TIM DE ZeEuw: Sterrewacht Leiden, Huygens Laboratorium, Wassenaarseweg 78, 2300 RA Leiden, The Netherlands

DAvid MerRITT: National Radio Astronomy Observatory, Edgemont Road, Charlottesville, VA 22901 